OPEN ACCESS

Edited by:

Clement Adebajo Meseko, National Veterinary Research Institute (NVRI), Nigeria

Reviewed by:

Julià Blanco, IrsiCaixa, Spain Nathalie Chazal, Université de Montpellier, France

*Correspondence: Dhruv Sareen dhruv.sareen@cshs.org

Vaithilingaraja Arumugaswami varumugaswami@mednet.ucla.edu

${ }^{\dagger}$ These authors have contributed equally to this work

Specialty section: This article was submitted to Virus and Host a section of the journal Frontiers in Cellular and Infection Microbiology

Received: 09 March 2021 Accepted: 21 May 2021 Published: 23 June 2021

Citation:

Shaharuddin SH, Wang V, Santos RS, Gross $A$, Wang $Y$, Jawanda $H$, Zhang Y, Hasan W, Garcia G Jr., Arumugaswami V and Sareen D (2021) Deleterious Effects of SARS-CoV-2 Infection on Human Pancreatic Cells. Front. Cell. Infect. Microbiol. 11:678482. doi: 10.3389/fcimb.2021.678482

\section{Deleterious Effects of SARS-CoV-2 Infection on Human Pancreatic Cells}

\author{
Syairah Hanan Shaharuddin ${ }^{1,2 t}$, Victoria Wang ${ }^{1,2 t}$, Roberta S. Santos ${ }^{1,2 t}$, \\ Andrew Gross ${ }^{1,2}$, Yizhou Wang ${ }^{3,4}$, Harneet Jawanda ${ }^{5}$, Yi Zhang ${ }^{5}$, Wohaib Hasan ${ }^{3,5,6}$, \\ Gustavo Garcia Jr. ${ }^{7}$, Vaithilingaraja Arumugaswami ${ }^{7,8^{*}}$ and Dhruv Sareen ${ }^{1,2,3,9 *}$

\begin{abstract}
1 Board of Governors Regenerative Medicine Institute, Cedars-Sinai Medical Center, Los Angeles, CA, United States, Biomedical Sciences, Cedars-Sinai Medical Center, Los Angeles, CA, United States, ${ }^{4}$ Genomics Core, Cedars-Sinai Medical Institute (SOCCI), Cedars-Sinai Medical Center, Los Angeles, CA, United States, ${ }^{6}$ Department of Pathology and Laboratory Pharmacology, David Geffen School of Medicine, University of California, Los Angeles, Los Angeles, CA, United States, ${ }^{8}$ Eli and Edythe Broad Center of Regenerative Medicine and Stem Cell Research, University of California, Los Angeles, Los Angeles, CA, United States, ${ }^{9}$ iPSC Core, David and Janet Polak Foundation Stem Cell Core Laboratory, Cedars-Sinai Medical Center, Los Angeles, CA, United States
\end{abstract} \\ ${ }^{2}$ Cedars-Sinai Biomanufacturing Center, Cedars-Sinai Medical Center, Los Angeles, CA, United States, ${ }^{3}$ Department of \\ Center, Los Angeles, CA, United States, ${ }^{5}$ Biobank and Translational Research Core, Samuel Oschin Comprehensive Cancer \\ Medicine, Cedars-Sinai Medical Center, Los Angeles, CA, United States, ${ }^{7}$ Department of Molecular and Medical
}

COVID-19 pandemic has infected more than 154 million people worldwide and caused more than 3.2 million deaths. It is transmitted by the Severe Acute Respiratory Syndrome Coronavirus 2 (SARS-CoV-2) and affects the respiratory tract as well as extra-pulmonary systems, including the pancreas, that express the virus entry receptor, AngiotensinConverting Enzyme 2 (ACE2) receptor. Importantly, the endocrine and exocrine pancreas, the latter composed of ductal and acinar cells, express high levels of ACE2, which correlates to impaired functionality characterized as acute pancreatitis observed in some cases presenting with COVID-19. Since acute pancreatitis is already one of the most frequent gastrointestinal causes of hospitalization in the U.S. and the majority of studies investigating the effects of SARS-CoV-2 on the pancreas are clinical and observational, we utilized human iPSC technology to investigate the potential deleterious effects of SARSCoV-2 infection on iPSC-derived pancreatic cultures containing endocrine and exocrine cells. Interestingly, iPSC-derived pancreatic cultures allow SARS-CoV-2 entry and establish infection, thus perturbing their normal molecular and cellular phenotypes. The infection increased a key cytokine, CXCL12, known to be involved in inflammatory responses in the pancreas. Transcriptome analysis of infected pancreatic cultures confirmed that SARS-CoV-2 hijacks the ribosomal machinery in these cells. Notably, the SARS-CoV-2 infectivity of the pancreas was confirmed in post-mortem tissues from COVID-19 patients, which showed co-localization of SARS-CoV-2 in pancreatic endocrine and exocrine cells and increased the expression of some pancreatic ductal stress response genes. Thus, we demonstrate that SARS-CoV-2 can directly infect human iPSC-derived pancreatic cells with strong supporting evidence of presence of the virus in post-mortem pancreatic tissue of confirmed COVID-19 human cases. This novel model of iPSC-derived pancreatic cultures will open new avenues for the comprehension 


\section{of the SARS-CoV-2 infection and potentially establish a platform for endocrine and} exocrine pancreas-specific antiviral drug screening.

Keywords: COVID-19, SARS-CoV-2, iPSCs, pancreas, acinar cells, ductal cells, islets, pancreatitis

\section{INTRODUCTION}

The novel coronavirus disease 2019 (COVID-19), caused by SARS-CoV-2 (Severe Acute Respiratory Syndrome Coronavirus2 ), initiated in China at the end of 2019 and rapidly escalated to global outbreak, infecting more than 154 million people and resulting in related fatalities in more than 3.2 million by beginning of May 2021 (JHU Dataset 2021). Although predominantly considered a respiratory disease, nearly one quarter of COVID-19 patients present other symptoms not related to the respiratory tract but to the gastrointestinal (GI) system (Cevik et al., 2020; Liu et al., 2020). SARS-CoV-2 enters host cells through binding of virus Spike protein (S) to ACE2 (Angiotensin-Converting Enzyme 2) receptor and activation of the virus by TMPRSS2 (Transmembrane Protease, serine 2). Interestingly, many other cells besides lung alveolar epithelial cells express ACE2 receptors, including heart, pancreas, GI tract, kidney, testis and other organs (Liu et al., 2020), thus making them a target for the virus. This is supported by studies demonstrating that virus can affect other tissues, including the heart (Sharma et al., 2020), vascular endothelial cells (Varga et al., 2020), kidney (Pelayo et al., 2020), liver (Amin, 2020) and the pancreas (Mukherjee et al., 2020; Pinte and Baicus, 2020; Tuttolomondo et al., 2020; Müller et al., 2021).

The pancreas, specifically the exocrine compartment (acinar and ductal cells), has high expression of ACE2 as analyzed by bulk RNA-seq data (GTEx database) and single-cell RNA-seq of the pancreas (NCBI-GEO database) (Liu et al., 2020). GTEx and TCGA (Genomic Data Commons Data Portal) datasets from human pancreas indicate no differences in ACE2 expression between males or females, as well as between younger (age $\leq 49$ years) and older (age $\geq 49$ years) populations (Li et al., 2020). In addition, recent findings have shown protein expression of ACE2 and TMPRSS2 in pancreatic cells from healthy humans, both in the endocrine and exocrine pancreas (Coate et al., 2020; Kusmartseva et al., 2020; Müller et al., 2021). Furthermore, few clinical reports provide evidence that some patients infected with SARS-CoV-2 who never presented pancreatic injuries show signs of acute pancreatitis-like symptoms, including elevated levels of serum amylase and lipase, and enlargement of the pancreas as seen in imaging evaluation (Kumaran et al., 2020; Liu et al., 2020). SARS-CoV-2 infection of the pancreas is certainly plausible as pancreatic ductal, acinar and islet cells express ACE2, so the virus could spread from the duodenal epithelium to the pancreas duct and then to acinar and islet cells. In fact, the virus was isolated easily from stool and from a pseudocyst of a COVID-19 patient with acute pancreatitis (de-Madaria and Capurso, 2021).

Acute pancreatitis is the major GI cause of hospitalization in the U.S.A., and despite the most common etiologies of this disease being related to gallstones and alcohol abuse, $10 \%$ of the cases are caused by infectious microorganisms including viruses (Rawla et al., 2017). Pertinent to the endocrine pancreas, it is now well-established that patients with type I (T1D) and II (T2D) diabetes have a higher risk for COVID-19 associated mortality, especially patients with associated comorbidities such as cardiovascular diseases and renal impairment, as indicated by a study with a large cohort of diabetic patients infected with SARS-CoV-2 (Holman et al., 2020). Diabetic patients (mostly T2D) infected with SARS-CoV-2 presented worse outcomes compared to non-diabetic patients, such as admission to the ICU, mechanical ventilation requirement and higher mortality rates (Apicella et al., 2020). These results were sustained by the in vitro evidence where replication of SARS-CoV-2 was demonstrated within pancreatic islet cultures, which led to impaired glucose-stimulated insulin secretion (Müller et al., 2021). Nevertheless, there is a paucity of knowledge whether and how SARS-CoV-2 can infect and impact the pancreas. This highlights the need for additional investigations to dissect and understand the potential influence of SARS-CoV-2 on human endocrine and exocrine pancreas.

Since primary human pancreatic cells are largely inaccessible and established cell lines do not fully represent human pancreas pathophysiology, we have developed novel methods to generate pancreatic progenitors from human induced pluripotent stem cells (iPSCs), which can be differentiated into endocrine (islet $\beta$ cells) and exocrine (acinar and ductal) cells. These cultures contain cells that are representative of human endocrine pancreas expressing endocrine NKX6.1 and C-peptide, exocrine acinar Amylase and Chymotrypsin (CTRC) and exocrine ductal Cytokeratin19 (CK19) and SOX9 cells. We show that iPSC-derived pancreatic cells including endocrine and exocrine cell types allow SARS-CoV-2 entry and establish infection, resulting in morphological perturbations as well as impaired expression of key markers. Importantly, these cellular phenotypes corresponded with inflammatory signatures. Infection of pancreatic tissue was also confirmed in postmortem pancreatic tissues from COVID-19 patients. Thus, these results suggest the pancreatic cells can be directly infected by SARS-CoV-2. The iPSC-based model described here provides a valuable novel platform for understanding the pancreas-specific cellular responses to SARS-CoV-2 as well as for antiviral drug development against SARS-CoV-2.

\section{METHODS}

\section{Human iPSC Culture}

The induced pluripotent stem cell (iPSC) line, CS0007iCTR-n7, utilized in this study was generated from a healthy volunteer at the iPSC Core at Cedars-Sinai Medical Center from the peripheral 
blood mononuclear cells (PMBCs) utilizing non-integrating oriP/ EBNA1-based episomal plasmid vectors, as described in (Rajamani et al., 2018). This approach results in highly cytogenetically stable iPSCs as tested by G-band karyotyping. All undifferentiated iPSCs were maintained in $\mathrm{mTeSR}^{+}$media (StemCell Technologies, Cat 05825) onto BD Matrigel ${ }^{\mathrm{TM}}$ matrix-coated plates.

\section{Generation of iPSC-differentiated Pancreatic Progenitors.}

iPSCs were single-cell dissociated using Accutase and plated onto Matrigel-coated plates at a density of $300,000 \mathrm{cells} / \mathrm{cm}^{2}$ using $\mathrm{mTeSR}^{+}$and $10 \mu \mathrm{M}$ Rho kinase Inhibitor (Stem Cell Tech). The following day, cells were directed into Definitive Endoderm (DE) using Phase I medium, which was composed of base medium MCDB 131 (Fisher Sci) supplemented with $100 \mathrm{ng} / \mathrm{ml}$ Activin A (R\&D), $2 \mu \mathrm{M}$ CHIR99021 (Stemgent), and $10 \mu \mathrm{M}$ Rho kinase Inhibitor (Stem Cell Tech.) for 1 day. For the next two days, the same base medium was used, but supplemented instead with $100 \mathrm{ng} /$ $\mathrm{mL}$ Activin A and $5 \mathrm{ng} / \mathrm{mL}$ FGF2 (Peprotech). Following this phase, cells were directed to form Posterior Foregut (PFG) using Phase II medium, which was composed of the same base medium as Phase I but supplemented with $50 \mathrm{ng} / \mathrm{mL}$ FGF10 (Peprotech), $0.25 \mu \mathrm{M}$ CHIR99021 and $50 \mathrm{ng} / \mathrm{ml} \mathrm{Noggin} \mathrm{(Peprotech),} \mathrm{for} 2$ days. To reach a Pancreatic Progenitor (PP) stage, cells were fed with Phase III medium, which was composed of DMEM supplemented with 50 ng/mL Noggin, 50 ng/mL FGF10, $2 \mu$ M Retinoic Acid (Sigma), and $0.25 \mu \mathrm{M}$ SANT1 (Sigma), for four days. More details about base medium formulation are described at Supplementary Table 1. This protocol was based on a published protocol to differentiate iPSCs into Pancreatic Progenitors (Memon et al., 2018).

\section{Generation of iPSC-Differentiated Pancreatic Endocrine (iPan ${ }^{\mathrm{ENDO}}$ ), Acinar (iPan ${ }^{\mathrm{EXO}}$ Acinar), and Ductal (iPan ${ }^{\mathrm{EXO}}$ Ductal) Cells}

To generate iPan ${ }^{\text {EXO }}$ Ductal cells, on Day 7 of differentiation, cells were single cell dissociated and seeded at $103.1 \mathrm{k}$ cells $/ \mathrm{cm}^{2}$ in Phase III medium supplemented with $10 \mu \mathrm{M}$ Rho kinase Inhibitor on a Matrigel-coated plate. From Day 8 to Day 26, cells were grown in $\mathrm{iPan}^{\mathrm{EXO}}$ Ductal phase media, which consisted of Phase III base medium supplemented with $25 \mathrm{ng} /$ $\mathrm{ml} \mathrm{FGF10,} 50 \mathrm{ng} / \mathrm{ml}$ EGF (Peprotech), and $25 \mathrm{ng} / \mathrm{ml}$ sDLL-1 (Peprotech). To generate iPan ${ }^{\text {ENDO }}$ and $\mathrm{iPan}^{\mathrm{EXO}}$ Acinar cells, based on Hohwieler et al. (2017) protocol, after 4 days of Phase III media, PPs were fed with Phase III base medium supplemented with $20 \mathrm{ng} / \mathrm{mL}$ FGF10, $1 \mu \mathrm{M}$ XXI (Sigma Aldrich), $50 \mathrm{ng} / \mathrm{mL}$ Noggin, $10 \mathrm{mM}$ Nicotinamide (SigmaAldrich), and $25 \mathrm{ng} / \mathrm{mL}$ Wnt3a (Peprotech) for seven days, daily feeding (Hohwieler et al., 2017). More details about base medium formulation are described at Supplementary Table 1.

\section{Generation and Maintenance of iPan ${ }^{\mathrm{EXO}}$ Organoid Cultures}

On the last day of Phase III, PPs were roughly dissociated via scraping and trituration. They were centrifuged at $170 \mathrm{x}$ G for 3 minutes, and then resuspended with a solution composed of
Phase III medium and Matrigel at a 1:4 ratio (1 of medium and 4 of Matrigel). $30 \mu \mathrm{L}$ of this solution with cells was plated into each well of a 96 round bottom plate and then incubated at $37^{\circ} \mathrm{C}$ for 20 minutes, before the plate was flipped upside down for 10 minutes. $100 \mu \mathrm{L}$ of Phase III base medium supplemented with 20 ng/mL FGF10, 50 ng/mL Noggin, $10 \mathrm{mM}$ Nicotinamide, and 25 $\mathrm{ng} / \mathrm{mL} \mathrm{Wnt} 3 \mathrm{a}$ was then added to the cells. Cells were fed every other day with the same media until Day 57 or when organoids contained lumen and were at least $150 \mu \mathrm{m}$ large.

\section{SARS-CoV-2 Stock}

SARS-CoV-2, isolate USA-WA1/2020, was obtained from the Biodefense and Emerging Infections (BEI) Resources of the National Institute of Allergy and Infectious Diseases (NIAID). Importantly, all studies involving SARS-CoV-2 infection of iPSC-derived pancreatic cells were conducted within a Biosafety Level 3 high containment facility at UCLA. SARSCoV-2 was passed once in Vero-E6 cells and viral stocks were aliquoted and stored at $-80^{\circ} \mathrm{C}$. Virus titer was measured in VeroE6 cells by TCID50 assay. Vero-E6 cells were cultured in DMEM growth media containing 10\% FBS, $2 \mathrm{mM}$ glutamine, pen/step, and $10 \mathrm{mM}$ HEPES. Cells were incubated at $37^{\circ} \mathrm{C}$ with $5 \% \mathrm{CO}_{2}$.

\section{SARS-CoV-2 Infection of iPSC-Derived Pancreatic (iPan) Cultures}

SARS-CoV-2 viral inoculum (MOI of 0.05 and 0.1 ) was prepared using acinar or ductal cell specific media. Human iPSCs were differentiated into iPSC-derived pancreatic (iPan) cultures containing iPan $^{\text {ENDO }}$, iPan ${ }^{\text {EXO }}$ Acinar and iPan ${ }^{\text {EXO }}$ Ductal cells in 96-well or 24-well plates before infection as detailed above, and the culture media at Day 26 of differentiation for iPan ${ }^{\text {EXO }}$ Ductal and Day 16 for iPan ${ }^{\text {EXO }}$ Acinar were replaced with $100 \mu \mathrm{l}$ of prepared inoculum. For mock infection, cell type specific media $(100 \mu \mathrm{l} /$ well $)$ alone was added. The inoculated plates were incubated for 1 hour at $37^{\circ} \mathrm{C}$ with $5 \% \mathrm{CO}_{2}$. At the end of incubation, the inoculum was replaced with fresh iPan ${ }^{\text {EXO }}$ Acinar or iPan ${ }^{\text {EXO }}$ Ductal culture medium. Cells remained at $37^{\circ} \mathrm{C}$ with $5 \% \mathrm{CO}_{2}$ for 24 hours (Day 1) or 72 hours (Day 3) before analysis. All studies involving active SARS-CoV-2 infection of iPSC-derived pancreatic cell cultures were conducted within a Biosafety Level 3 facility at University of California in Los Angeles (UCLA), CA, USA.

\section{Immunofluorescence and Imaging of Cells}

Cells subjected to immunofluorescence were fixed with $4 \%$ paraformaldehyde (PFA) in phosphate-buffered saline (PBS) for 20 minutes and subsequently washed with PBS. Fixed cells were then permeabilized and blocked for 1 hour in a blocking buffer containing PBS with 10\% donkey serum (Millipore) and $0.1 \%$ Triton-X (Bio-Rad). Primary antibodies were diluted in the blocking buffer and added to the cells overnight at $4^{\circ} \mathrm{C}$. The following primary antibodies and dilutions were used: SARS Coronavirus (1:400, NR-10361, BEI Resources), SARS-CoV-2 Spike S1 (1:100, 40150-R007, SinoBiological), eCAD (1:100, AF68, R\&D Systems), MIST1 (1:100, MA1517, Invitrogen), CK19 (1:100, PIMA512663, Thermofisher), SOX9 (1:250, 
AB5535, Millipore), ACE2 (1:100, AB15348, abcam), Chymotrypsin (1:100, MAB1476, Millipore), TMPRSS2 (1:250, AB92323, Abcam) and Amylase (1:100, A8273, Sigma). After washing using PBS with $0.1 \%$ Tween-20 (ThermoFisher), cells were incubated with appropriate species-specific Alexa Fluorconjugated secondary antibodies (ThermoFisher) diluted in a blocking buffer $(1: 1,000)$ for 1 hour at room temperature. After washing in PBS with $0.1 \%$ Tween-20, cells were incubated in Hoechst 33342 diluted in PBS (1:2,500) for $15 \mathrm{~min}$. Immunofluorescence images were visualized using appropriate fluorescent filters using ImageXpress Micro XLS (Molecular Devices) and analyzed using ImageJ Software. Image quantification was performed using CellProfiler Software (v3.1.9).

\section{Real-Time qPCR}

Relative gene expression was quantified using RT-qPCR. For this, cells were washed with PBS to remove any possible remaining viral inoculum and the total RNA was isolated with RLT (Qiagen). RNA was then extracted with RNeasy Micro Kit (Qiagen) according to the manufacturer's instructions. The concentration of RNA was determined by spectrophotometric analysis (Qubit 4 Fluorometer, ThermoFisher) and the purity with NanoDrop (ThermoFisher); all samples had a $\mathrm{A}_{260 / 280}$ ratio around 2.0 (Desjardins and Conklin, 2010). After, RNA (1 $\mu \mathrm{g})$ was reverse transcribed to cDNA with oligo(dT) using the High Capacity cDNA Reverse Transcription kit (ThermoFisher). Realtime qPCR was performed in triplicates using SsoAdvanced Universal SYBR Green Supermix (Biorad) and specific primer sequences to each gene (Supplementary Table 2), on a CFX384 Real Time system (Bio-Rad). Human RPL13 was used as the reference gene and relative expression was determined using $2^{-\Delta \Delta}$ Ct method.

\section{Post-Mortem Human Pancreatic Tissues a) Real-Time qPCR From Snap-Frozen Tissues}

Post-mortem pancreatic samples were isolated from the head of the pancreas (preferably) from patients that were infected with SARS-CoV-2 and passed from complications related to the disease (COVID-19 patients), or patients that were not infected with SARS-CoV-2 and passed from complications not related to it (Control patients). These samples were isolated 1-3 days after the patient's death and were snap-frozen in liquid nitrogen. Samples were stored for longer in $-80{ }^{\circ} \mathrm{C}$ before used for RNA extraction. For RNA isolation, Trizol (Thermo) was used and for RNA extraction, RNeasy Mini Kit (Qiagen) was used according to the manufacturer's instructions. The concentration of RNA was determined by spectrophotometric analysis (Qubit 4 Fluorometer) and the purity with NanoDrop, as described above; all samples had a $\mathrm{A}_{260 / 280}$ ratio around 2.0. After, RNA $(2 \mu \mathrm{g})$ was reverse transcribed to cDNA with oligo(dT) using the High Capacity cDNA Reverse Transcription kit. Real-time qPCR was performed in triplicates using SsoAdvanced Universal SYBR Green Supermix (Bio-Rad) and specific primer sequences to each gene can be found in Supplementary Table 3. qPCR was performed on a CFX384 Real Time system. Human $\beta$-ACTIN was used as the reference gene and relative expression was determined using $2^{-\Delta \Delta} \mathrm{Ct}$ method.

\section{b) Immunohistochemistry From Paraffin Embedded Tissues}

Pancreatic tissues were fixed in $10 \%$ formalin and then paraffin embedded. Blocks were sectioned at $4 \mu \mathrm{m}$ thickness and mounted at microscope slides (Superfrost Plus microscope slides, Fisher). Slides were washed $2 \mathrm{x}$ in Xylene for 10 minutes each. This was followed by $2 \mathrm{x} 5$-minute washes with $100 \%$ ethyl alcohol, 1x 3-minute wash with 95\% ethyl alcohol, and 1x 3minute wash with $75 \%$ alcohol for rehydration. They were then washed $3 \mathrm{x}$ with PBS for 5 minutes before beginning antigen retrieval. Samples were submerged in $10 \mathrm{mM}$ pH 6.0 sodium citrate Buffer, and then microwaved for 10 minutes at $80 \%$ power. Once cooled at room temperature for one hour, they were washed $3 \mathrm{x}$ for 5 minutes each with PBS. Samples were blocked for 2 hours in the same blocking buffer as mentioned above (PBS with 10\% donkey serum and $0.1 \%$ Triton-X). Samples were incubated $4^{\circ} \mathrm{C}$ overnight with primary antibodies in the same concentrations as mentioned above. The following day, they were washed $3 \mathrm{x}$ for 10 minutes each in PBS, incubated one hour at room temperature with the secondary antibodies at concentrations of 1:1000, and then finally washed $3 \mathrm{x}$ for 10 minutes each before mounted with ProLong ${ }^{\mathrm{TM}}$ Gold Antifade Mountant with DAPI (Invitrogen).

\section{RNA Sequencing (RNASeq)}

\section{a) RNA Extraction and Sequencing}

RNA was extracted from pelleted cells using RNeasy Micro Kit (Qiagen) and was prepared for sequencing with the Illumina TruSeq Stranded mRNA library preparation kit (Illumina, San Diego, CA) by the Cedars-Sinai Applied Genomics, Computation, and Translational Core. Concentration and quality of RNA was assessed on a Qubit fluorometer (ThermoFisher Scientific, Waltham, MA) and 2100 Bioanalyzer (Agilent Technologies, Santa Clara, CA) respectively. Complementary DNA was reverse transcribed using Invitrogen's Reverse Transcriptase kit (Carlsbad, CA) and converted into double-stranded DNA (dsDNA). The dsDNA was then enriched using PCR before purification with Agencourt AMPure XP beads (Beckman Coulter, Brea, CA). The enriched purified DNA was then quantified and resolved via Qubit and Bioanalyzer. Sample libraries were then multiplexed and sequenced on Illumina's NextSeq 500 platform (San Diego, CA) using 75 bp single-end sequencing.

\section{b) Analysis}

Raw reads were quantified by mapping them with the STAR aligner (version 2.5.0) (Dobin et al., 2013)/RSEM (version 1.2.25) (Li and Dewey, 2011) to the GRCh38 human reference transcriptome based on human GENCODE version 33 (www. gencodegenes.org) as well as the GenBank: MT246667.1 SARS$\mathrm{CoV}-2$ reference viral genome. Expression tables were postprocessed with a custom $\mathrm{R}$ script that filtered out non-coding RNA based on the transcript biotype assigned by biomaRt. Initial analyses were performed on the BioJupies platform. Principal 
Component Analysis was then performed in $\mathrm{R}$ using the prcomp package. Differential expression tables were calculated in $\mathrm{R}$ using the DESeq2 package. Differential expression tables were used to plot heatmaps and volcano plots in ggplot. Enrichment analysis was performed by uploading the top 500 upregulated and downregulated transcripts to the Enrichr gene enrichment analysis portal (Chen et al., 2013; Kuleshov et al., 2016). Enrichment results of interest (including Gene Ontology enrichment and COVID-related gene enrichment) were exported from Enrichr as text files and imported into $\mathrm{R}$ for plotting using ggplot. All analysis and plotting scripts in $\mathrm{R}$ are available on github.com at github.com/Sareen-Lab/COVID. Gene transcript tables as well as the original FASTQ files are available through NCBI's GEO database at accession number GSE165890.

\section{Statistical Analyses}

Data are presented as mean \pm standard error of the mean (SEM). Statistical significance between groups was determined by Oneway ANOVA followed by Dunnet post-test. Two-tailed paired Student's test was used as appropriate. $P$ values $<.05$ were considered statistically significant. Statistical analyses and graphs were generated using GraphPad Prism 7 for Windows Software (GraphPad Software).

\section{RESULTS}

\section{iPSC-Derived Pancreatic Cells Exhibit ACE2 and TMPRSS2 Expression}

Human iPSCs were differentiated into pancreatic adherent (2D) and organoid (3D) cultures. Protocols for differentiation after the pancreatic progenitor stage were directed to bias the cell fate of the cultures containing either exocrine acinar cells (iPan ${ }^{\mathrm{EXO}}$ Acinar) or exocrine ductal cells (iPan ${ }^{\text {EXO }}$ Ductal). iPan ${ }^{\text {EXO }}$ Acinar cultures express some markers that are characteristic of mature acinar cultures such as cytoplasmic staining of digestive enzymes Amylase (AMY) and Chymotrypsin C (CTRC), and greater staining of nuclear transcription factor MIST1, which is characteristic of acinar cells that are not fully mature. In these cultures, cells that are $\mathrm{AMY}^{-}$are $\mathrm{MIST}^{+}$, which indicates we have mixed cultures of acinar progenitor and mature cells. Acinar cultures also contain some C-peptide expressing endocrine islet $\beta$-cells (iPan ${ }^{\text {ENDO}}$ ). iPan ${ }^{\text {EXO }}$ Ductal cultures express cytoskeletal staining of Cytokeratin 19 (CK19). Interestingly, both $\mathrm{AMY}^{+}$iPan $^{\text {EXO }}$ Acinar and CK19 ${ }^{+}$iPan $^{\text {EXO }}$ Ductal cultures exhibit ACE2 and TMPRSS2 protein expression (Figures 1A, B), which play a critical part in allowing SARSCoV-2 cell entry (Hoffmann et al., 2020). Notably, while coexpression is seen, not all C-peptide ${ }^{+}$cells express ACE2 (Figure 1C), and this seems to be consistent with current literature, which shows lower ACE2 expression in endocrine cells (Coate et al., 2020; Kusmartseva et al., 2020). ACE2 and TMPRSS2 gene expression was also measured in iPSC-derived pancreatic cultures. As a negative control, pluripotent iPS cells were used, while cadaveric pancreatic acinar tissues, human pancreatic duct epithelium cell line $\mathrm{H} 6 \mathrm{C} 7$, and lung tissues from healthy donors were used as positive controls. Human iPSC-derived neurons were also included as the brain-derived neural tissue lacks ACE2 expression (Lonsdale et al., 2013; Carithers et al., 2015). The results show that cadaveric pancreatic acinar tissues and immortalized human pancreatic duct epithelium cell line $\mathrm{H} 6 \mathrm{C} 7$ have high ACE2 expression. Relative to iPSCs, iPan ${ }^{\text {EXO }}$ Acinar and iPan ${ }^{\text {EXO }}$ Ductal cultures also contain significantly higher levels of ACE2. There is also a significantly higher expression of TMPRSS 2 in iPan ${ }^{\text {EXO }}$ Acinar and human acinar tissues, with low levels in iPan ${ }^{\text {EXO }}$ Ductal cells (Figure 1D). It is known that occasionally different normal and diseased tissues can be differently correlated in their protein and gene expression and there can be some discordance between protein and gene expression for a subset of genes within pancreas (Kosti et al., 2016). Differentiated iPan ${ }^{\text {EXO }} 3 \mathrm{D}$ organoids also show co-localization of ACE2 protein along with acinar and ductal markers (Supplementary Figure 1).

\section{SARS-CoV-2 Can Directly Infect iPan ${ }^{\text {EXO }}$ Ductal Cultures and Elicit Abnormal Cellular Phenotypes}

After 26 days of pancreatic ductal differentiation starting from iPSCs, cultures were infected with SARS-CoV-2 at a multiplicity of infection (MOI) 0.05 and 0.1 , and cells were collected for analysis after one day (Day 1) or after three days (Day 3) (Figure 2A). These MOIs allowed for establishing de novo infection and active viral replication for the viral kinetics studies. The mock condition was treated with ductal media with no virus. MOI 0.05 infection shows $4 \%$ and $19 \%$ of cells infected with SARS-CoV-2 on Day 1 and Day 3 respectively, while MOI 0.1 infection shows $10 \%$ and $19 \%$ of infected cells, respectively (Figure 2B), showing viral infectivity increased from Day 1 to Day 3 in both MOIs. Although more cells got infected from Day 1 to Day 3, less cells were observed in culture comparing infected cultures with uninfected cultures, which suggests that the virus possibly decreased cell viability (data not shown). As expected, the mock condition has negative SARSCoV-2-Spike S1 staining (Figure 2B). RT-qPCR for SARS-CoV2 nucleocapsid gene $N 1$ shows SARS-CoV-2 mRNA production increased in the infected conditions compared to mock condition as seen in Figure 2C. Thus, iPSC-derived pancreatic cultures allow SARS-CoV-2 entry and establish infection.

SOX9 is a well-established nuclear transcription factor involved in determining ductal specification from pancreatic progenitors. Interestingly, SARS-CoV-2 positive cells in the infected iPan ${ }^{\mathrm{EXO}}$ Ductal cultures at both MOIs show a more enlarged cellular morphology as observed by CK19 and SOX9 staining pattern (Figure 3A) and abnormal cytoplasmic SOX9 localization instead of the typical nuclear localization observed in normal ductal cells as evident in the mock condition and uninfected cells (Figure 3B). In fact, we observe a higher number of ductal cells with mislocalization of SOX9 in the cytoplasm of infected cells at both Day 1 and Day 3 compared with mock cells, and both MOIs seem to have a similar pattern of mislocalization (no statistical difference between MOIs in both 
A

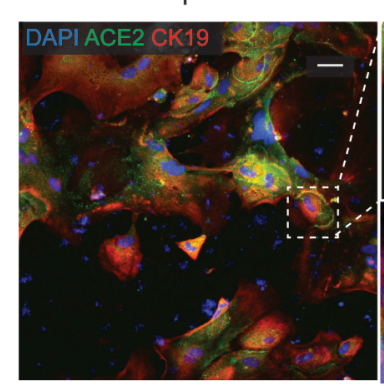

i

ii
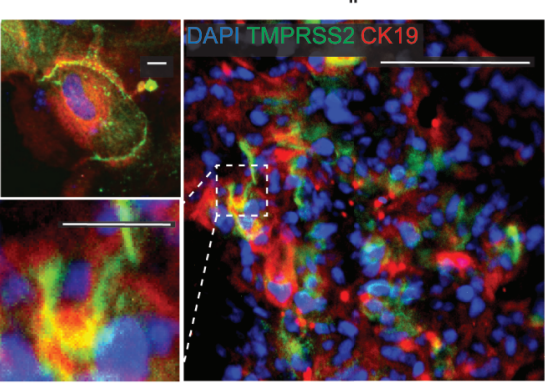

B
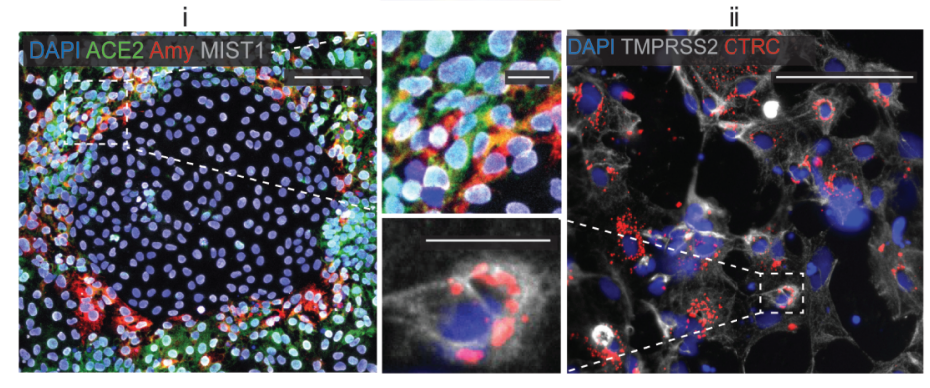

C
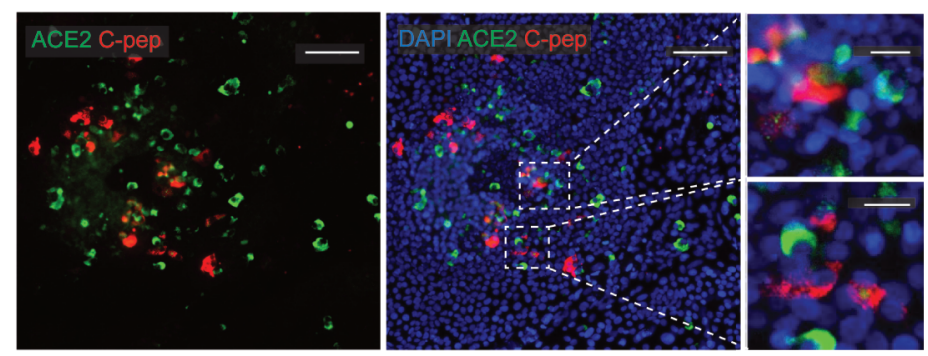

D
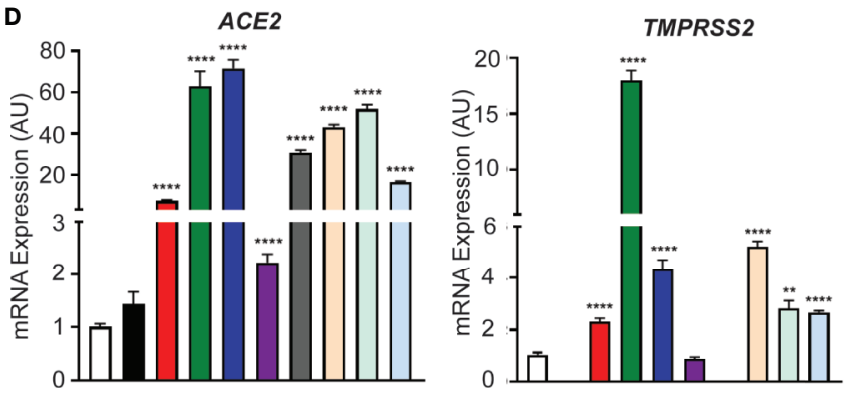

$\square$ IPSC

- iPSC-derived neuron

[ iPan ${ }^{\mathrm{EXO}}$ Acinar

[ Human acinar tissue 1

Human acinar tissue 2

¿ iPan ${ }^{\mathrm{EXO}}$ Ductal

口 $\mathrm{H} 6 \mathrm{C} 7$ ductal cell line

C. Human lung tissue 1

C Human lung tissue 2

Human lung tissue 3

FIGURE 1 | Expression of ACE2 and TMPRSS2 in exocrine pancreatic cells. (A) iPan EXO Ductal cells CK19 (red) exhibiting i. ACE2 (green) and ii. TMPRSS2 (green) expression. (B) $\mathrm{PPan}^{\mathrm{EXO}}$ Acinar cells Amylase (red), MIST1 (gray) exhibit i. ACE2 (green) expression and ii. Pan ${ }^{\mathrm{EXO}}$ Acinar CTRC (red) exhibit TMPRSS2 (green). (C) $\mathrm{PPan}^{\mathrm{EXO}}$ Acinar and $\mathrm{Pan}{ }^{\mathrm{ENDO}}$ cultures contain some endocrine C-peptide expressing cells, which also co-stain with ACE2. (D) iPSC-derived pancreatic exocrine cells as well as human acinar tissues and human ductal cell line H6C7 express ACE2 and TMPRSS2. Data is shown as mean \pm SEM with statistical significance determined by unpaired two-tailed t-test. ${ }^{* *} \mathrm{p}<0.01$ and ${ }^{{ }^{* \star *} \mathrm{p}}<0.0001$. Scale bar represents $100 \mu \mathrm{m}$, and $20 \mu \mathrm{m}$ for zoomed panels adjacent to main images. ICC images shown here are representative results from 27 independent sites acquired. RNA was extracted from aggregates of 2-3 biological replicates (using 12 wellplates), and GPCR was run with 3 technical replicates per sample. These results were pooled from 2 independent rounds of infection experiments.

Day 1 and Day 3) (Figure 3C). Meanwhile, CK19 is present across all treatments and timepoints, with no obvious differences in staining localization of the infected population. ACE2 expression is observed in Day 3 cells with no distinct differences between mock and MOI 0.05 and 0.1 conditions (Supplementary Figure 2). $\mathrm{CK} 19^{+}$ductal cells become multinucleated when infected with SARS-CoV-2 (MOI 0.1), while uninfected ductal cells remain mononucleated (Mock) (Figure 3D). This phenomenon has been described as syncytia formation, the fusion of multiple mononucleated cells, and known to amplify apoptotic signals in response to viral infection (Scheller and Jassoy, 2001; Salsman et al., 2005; Nardacci et al., 2015). In MOI 0.1 treatment of Day 3 culture, the percentage of multinucleated infected ductal cells is $81.6 \%$, 


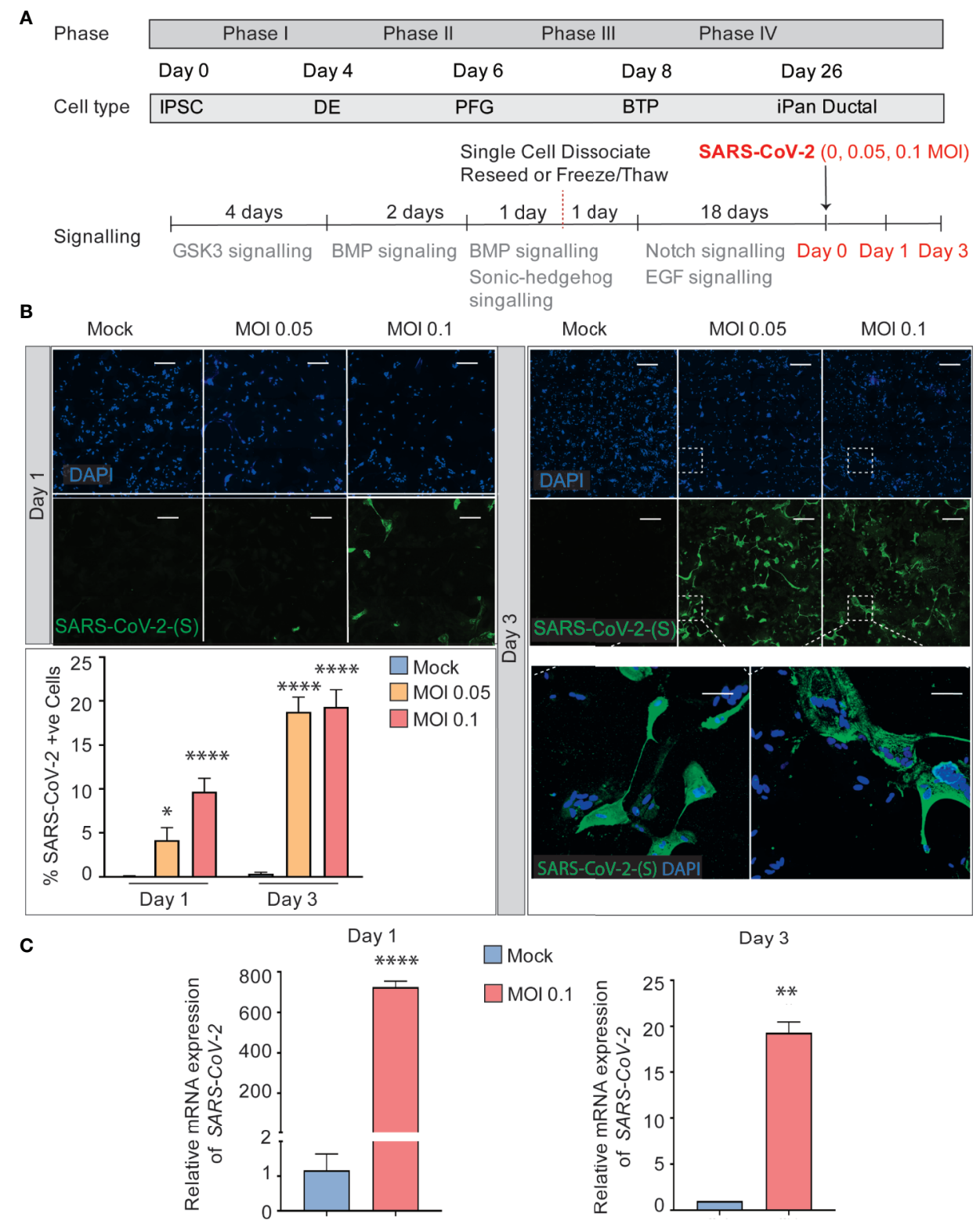

FIGURE 2 | SARS-CoV-2 can infect iPan ${ }^{\mathrm{EXO}}$ Ductal Cells. (A) Differentiation scheme of pancreatic ductal cells and infection of SARS-CoV-2 on Day 26.

(B) Immunocytochemistry staining of SARS-(S) at Day 1 and Day 3 (Scale bar is $200 \mu \mathrm{m})$. (C) RT-qPCR showing upregulation of SARS-CoV-2 N1 from mock to infected cells at different days. Data is shown as mean \pm SEM with statistical significance determined by unpaired two-tailed t-test. ${ }^{*} p<0.05$, ${ }^{* *} p<0.01,{ }^{* \star \star *} p<0.0001$. Scale bar represents $200 \mu \mathrm{m}$. ICC images shown here are representative results from 36 independent sites acquired. RNA was extracted from aggregates of 6 -15 biological replicates (using 96 well-plates), and qPCR was run in 3 technical replicates per sample. These results were pooled from 2 independent rounds of infection experiments.

which is significantly higher than the percentage of multinucleated non-infected ductal cells, 12.2\% (Figure 3E).

\section{SARS-CoV-2 Can Infect iPan ${ }^{\text {EXO }}$ Acinar and iPan ${ }^{E N D O}$ Cells Resulting in Activation of Proinflammatory Genes}

iPan ${ }^{\text {EXO }}$ Acinar cultures containing predominantly Chymotrypsin $\mathrm{C}$ (CTRC) and Amylase (AMY) positive cells and few iPan ${ }^{\text {ENDO }}$ C-peptide positive islet $\beta$-cells were differentiated for 16 days before being infected with SARS-CoV-2 at a MOI of 0.1. A separate mock condition was cultured in parallel and not infected. Like the iPan ${ }^{\text {EXO }}$ Ductal cultures, infected and uninfected cultures were fixed or lysed after one day or three days of infection (Figure 4A). CTRC-positive cells, which have a granular morphology that likely reflects zymogen granule formation, showed direct infection by SARS-CoV-2 (Figure 4B). Interestingly, a subpopulation of SARS-CoV-2 positive cells in these cultures were also C-peptide positive 
(Supplementary Figure 3). On Day 1 post-infection, 0.63\% of total cells were infected, which increased to $1.12 \%$ by Day 3 . For both days, the difference in percentage of infected cells was statistically significant compared to mock condition (Figure 4C), which was similarly seen on mRNA level by RTqPCR for SARS-CoV-2 nucleocapsid gene N1, showing high
mRNA production of SARS-CoV-2 in the infected conditions (Figure 4D).

To investigate whether SARS-CoV-2 infection perturbed the inflammatory pathway, multiple genes known to be associated with pancreatitis-related inflammation were assessed using RTqPCR. Among those, the genes CXCL12, NFKB1, and STAT3

A

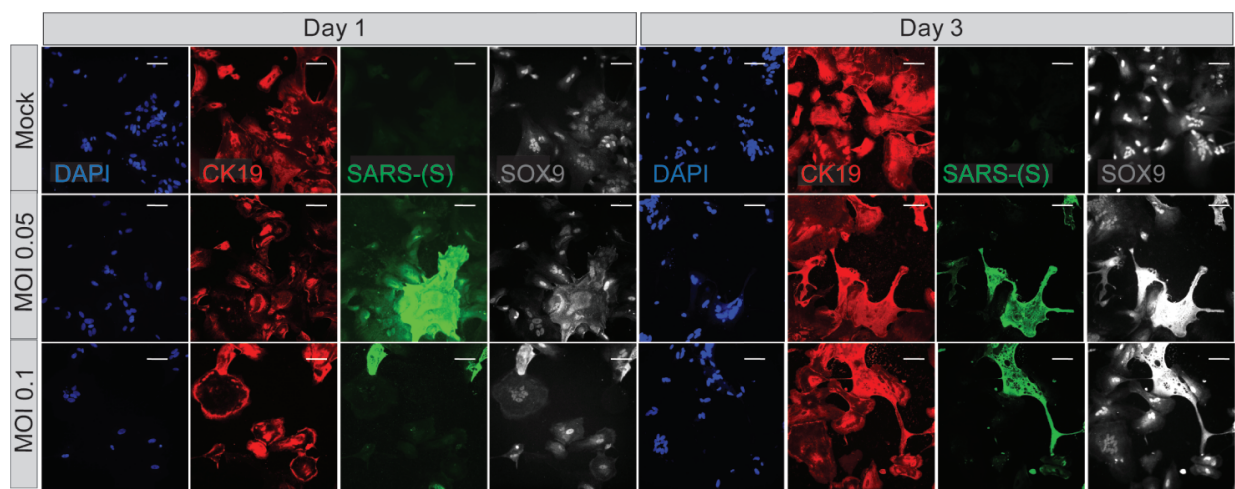

B

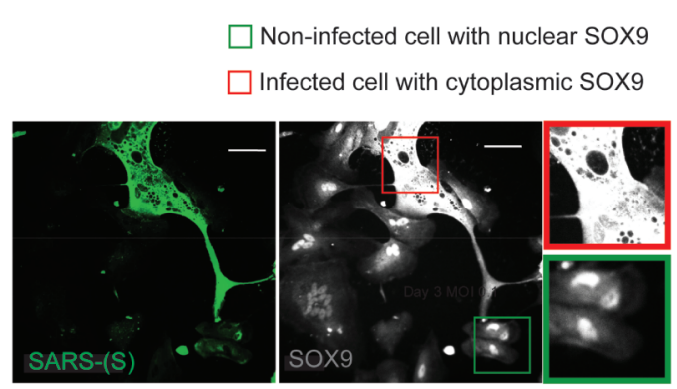

C

$\square$ Mock

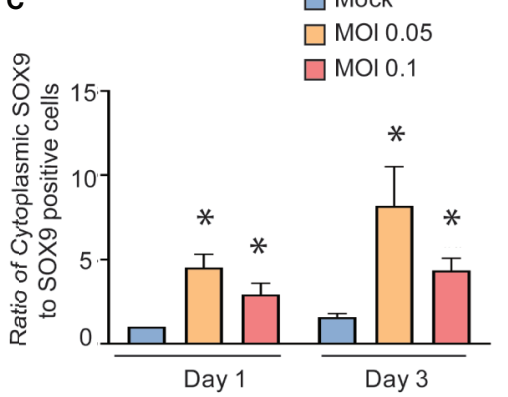

D

Day 3 iPan Ductal Cells MOCK

$\square$ Mononucleated SARS CK19+

E

$\square$ SARS CK $19^{+}$ MOI 0.1
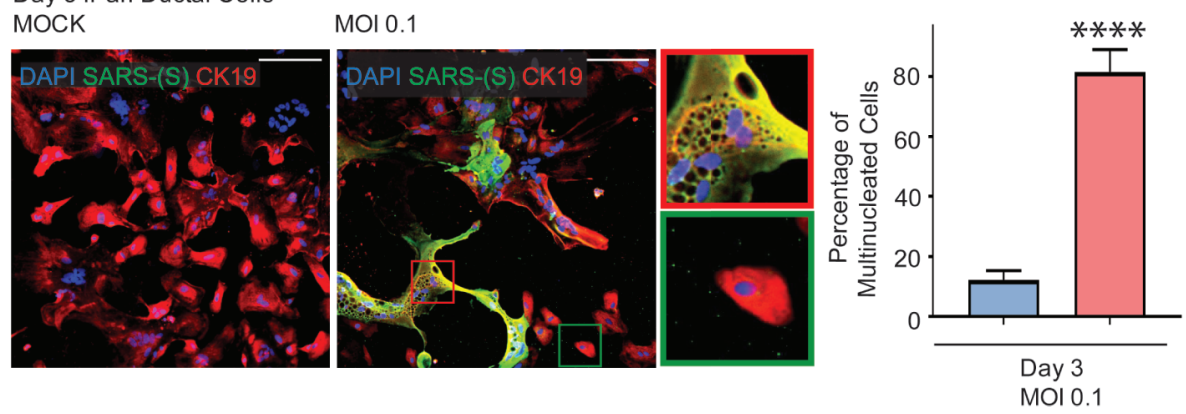

FIGURE 3 | SARS-CoV-2 elicits abnormal cellular phenotypes in iPan ${ }^{\mathrm{EXO}}$ Ductal cultures. (A) Day 1 and Day 3 of post infected iPan ${ }^{\mathrm{EXO}}$ Ductal cells. Immunocytochemistry staining of ductal cell markers CK19 (red), SOX9 (gray), and SARS-CoV-2 infected cells (green) on Day 1 and Day 3 with varying titers of virus (Mock, MOI 0.05, and 0.1). (B) SOX9 translocation in infected cells. (Scale bar is $200 \mu \mathrm{m}$ ). (C) Histogram is showing the ratio of cells with mis localized cytoplasmic SOX9 over total nuclear SOX9 positive cells in the culture, comparing mock vs SARS-CoV-2 infected ductal cultures at MOI 0.05 and 0.1 . Data is shown as mean \pm SEM with statistical significance determined by unpaired two-tailed t-test. ${ }^{*} \mathrm{p}<0.05$. Scale bar represents $200 \mu \mathrm{m}$. Zoomed panels adjacent to main images are $2.3 \mathrm{x}$ larger. ICC images shown here are representative results from 36 independent sites acquired. These results were pooled from 2 independent rounds of infection experiments. (D) Immunocytochemistry staining of SARS-(S) at Day 3 Mock and MOI 0.1. Zoom in panels indicate mononucleated SARS ${ }^{-}$CK19 $^{+}$cell (green box) and multinucleated SARS ${ }^{+} \mathrm{CK}_{1} 9^{+}$cell (red box). Scale bar represents $200 \mu \mathrm{m}$. ICC images shown here are representative results from 9 independent sites acquired. (E) Histogram is showing the percentage of multinucleated cells in non-infected SARS ${ }^{-}$CK $19^{+}$population and infected SARS ${ }^{+}$CK $19^{+}$population. Data is shown as mean \pm SEM with statistical significance determined by unpaired two-tailed t-test. ${ }^{\star \star \star \star} \mathrm{p}<0.0001$. 
showed significant upregulation at Day 3 in infected cells compared to mock condition (Figure 4E), while no significant change was observed on infected cells by Day 1 compared to the mock condition (Supplementary Figure 4). No significant change in expression was seen on other inflammatory markers, such as $I L 1 B$ and TNFA, on both days (Supplementary Figure 4).

\section{Transcriptional Analysis of SARS-CoV-2 Infected iPSC-Derived Pancreatic Cultures Demonstrates Viral and Pancreas-Specific COVID-19 Associated Disease Signatures}

The iPan cultures containing iPan ${ }^{\mathrm{EXO}}$ Acinar and $\mathrm{iPan}^{\mathrm{ENDO}}$ cells infected with 0.1 MOI SARS-CoV-2 were harvested on Day 1 and Day 3 post-infection for transcriptomic analysis after mRNA sequencing, as well as cells that were not infected (mock conditions). After mapping genomic reads in infected cultures, mapped reads were detected from the SARS-CoV-2 genome that confirm active SARS-CoV-2 viral replication within infected cultures (Supplementary Table 3). Principal component analysis (PCA) of Day 1 and Day 3 infected cultures demonstrated that $37.9 \%$ and $41.1 \%$ of the variance in the gene expression differences could be attributed to principal component 1 (PC1) in Day 1 and Day 3 infected cultures, respectively (Figure 5A). Both PCA and the heatmaps of differentially expressed genes clearly demonstrated transcriptomic clustering of samples in either the mock or infected condition in both days (Figure 5B). Upon further interrogation of the Day 3 data, SARS-CoV-2 infection induced significant pancreas-specific gene expression changes within iPSC-derived pancreatic cultures including upregulation of PDX1, INS, GCG, CHGA, CFTR, IGFBP7 and $G H R L$ and downregulation of genes involved in cellular secretory pathway such as GOLGA8A and GOLGA8B. Significant changes in expression of chemokine and immunomodulatory genes were detected. Most were upregulated such as PTGES, MIF, CCR7, CXCL6 and CXCL12, which encodes immune cytokines known to be transcriptionally upregulated during SARS-CoV infection. Similarly, genes reflecting pathogenic interaction and antiviral responses in host cells such as THOC1, TRIM28, CD37, TRAF3IP1, DDX17, NCBP3, HYAL2, were also perturbed (Supplementary Table 4). Gene enrichment analysis comparing the 500 genes most upregulated in infected day 3 samples to Gene Ontology's Biological Process database identified several biological processes that contained a high number of genes found among those upregulated in our infected sample. These included the "[Signal Recognition Particle]-dependent cotranslational protein targeting to membranes" process, the "protein targeting to ER" process, and several processes associated with viral infection (Figure 6A) consisting of mainly ribosomal complex large and small subunit genes. This is consistent with the idea that SARS-CoV-2 like many other viruses requires recruitment of a variety of host cell factors including ribosomal proteins to participate in viral protein biosynthesis, in order to survive, accumulate and propagate in the pancreatic cells. A gene enrichment analysis comparing the 500 most downregulated genes in our samples to Gene Ontology's cellular components data set found that the most significant cellular components that appear to be downregulated include nuclear body, nuclear specks and nucleoplasm (Figure 6B), which is also consistent with the downregulation of the specific nuclear pore complex (NPC) genes such as NPIPB3, 4, 5 (Figure 5C). Numerous viral pathogens have evolved different mechanisms to hijack the NPC in order to regulate trafficking of viral proteins, genomes and even capsids into and out of the nucleus thus promoting virus replication (Le Sage and Mouland, 2013). As expected, the cytosolic ribosomal units are the most significantly upregulated cellular component (Figure 6B). Performing the same gene enrichment analysis against NCBI's COVID-19 associated transcripts dataset available on the Enrichr database (Kuleshov et al., 2020) identified several dozen downregulated genes classified as downregulated by SARSCoV-2 in pancreatic organoids (Figure 6C). Taken together, these results indicate that SARS-CoV- 2 infection induces significant transcriptional changes within iPSC-derived pancreatic cultures.

\section{Post-Mortem Human Pancreatic Tissues From COVID-19 Patients Show Infectivity and Perturbed Expression of Pancreatic Genes}

Post-mortem human pancreatic samples were obtained from individuals who succumbed from complications related to COVID-19 infection (COVID-19 patients) or from those who were not infected by SARS-CoV-2 and were deceased due to complications unrelated to COVID-19 (Control patients). Samples were processed for immunohistochemistry of SARS$\mathrm{CoV}-2$-Spike S1 and pancreatic markers or processed for analysis of changes in gene expression. As shown in Figures 7A-C, SARSCoV-2-S was detected in pancreas of COVID-19 patients, demonstrating the susceptibility of the human pancreas to the virus. To better understand which cell types were infected by SARS$\mathrm{CoV}-2$ in the pancreas, cells were stained for specific endocrine and exocrine markers, such as Amylase and Chymotrypsin (CTRC) for acinar cells, Cytokeratin 19 (CK19) for ductal cells, and C-peptide for endocrine islet $\beta$-cells. Interestingly, many of the pancreatic cell types tested here co-localized with SARS-CoV-2-S. The virus was present in the majority of the $\mathrm{CTRC}^{+}$and some clusters of Amylase $^{+}$cells (Figures 7A, B). However, SARS-CoV-2-S was not detected in ductal $\mathrm{CK} 19^{+}$cells (Supplementary Figure 5) in the limited set of histology specimens from COVD19 patients examined. In the pancreatic endocrine compartment, interestingly, SARS-CoV-2 staining was frequently concentrated in C-Peptide ${ }^{+}$ islet clusters (Figure 7C).

To examine whether the infected pancreatic tissues from COVID-19 patients may have differential expression of specific genes, snap-frozen post-mortem tissues from COVID-19 patients and Control subjects were assessed changes in expression of pancreas-specific genes. Interestingly, mRNA expression of ductal markers was increased in the COVID-19 samples, such as KRT19, CFTR, CA2, and HNF1B as seen in Figure 7D. Other pancreatic and inflammatory markers were also tested and although showed trends of perturbations, they were not statistically different between the groups likely due to 
A

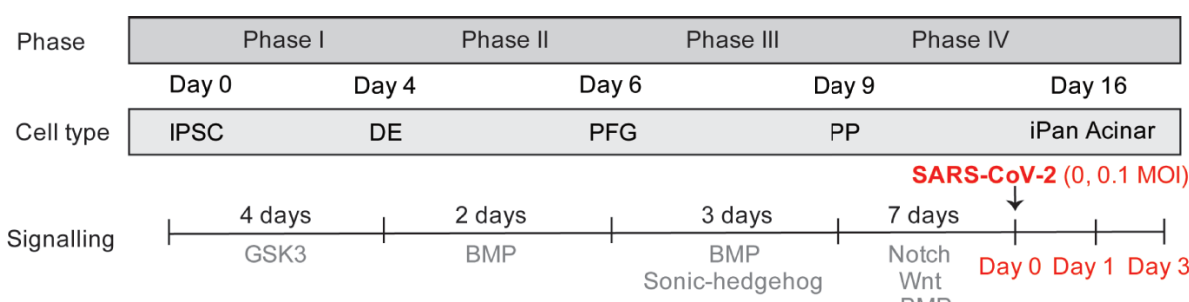

B
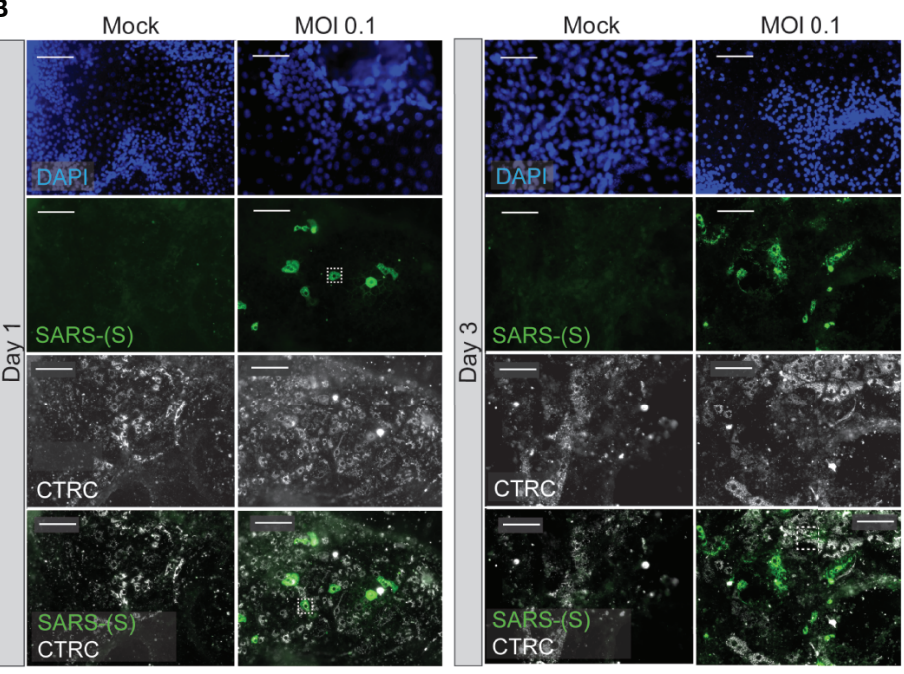

Day

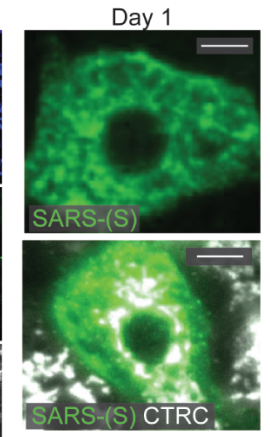

Day 3

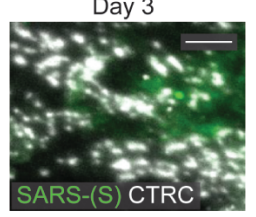

C

D

E
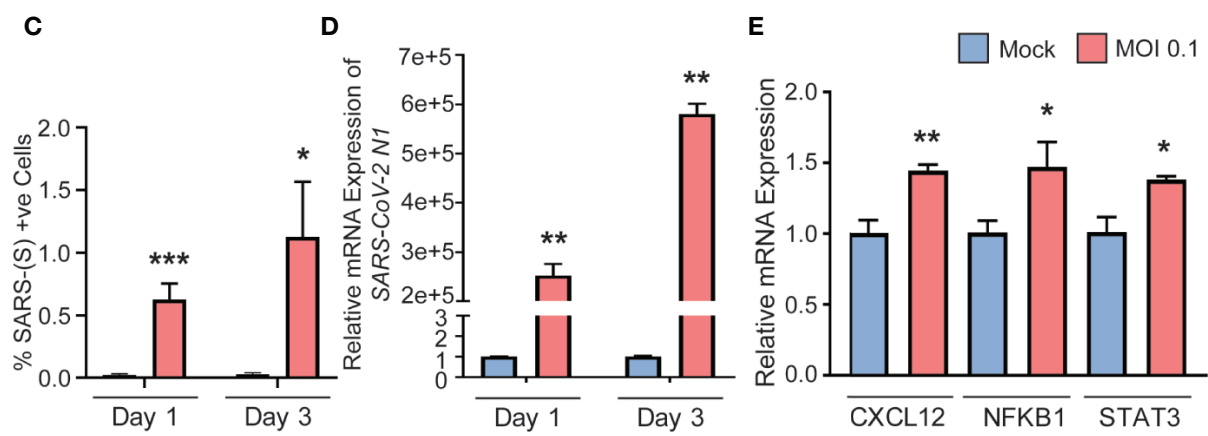

FIGURE $4 \mid \mathrm{iPan}{ }^{\mathrm{EXO}}$ Acinar cultures can be infected with SARS-CoV-2 and result in upregulation of some proinflammatory genes. (A) Differentiation and infection timeline for the iPan ${ }^{\mathrm{EXO}}$ Acinar cells. Cells were grown 16 days before being infected. Cells were fixed or lysed on the first and third days of infection.

(B) Immunocytochemistry staining of SARS-(S) on Day 1 and 3 of mock and infected cells. (C) Quantification of immunocytochemistry images shows significant increase in SARS-(S) positive cells in the population treated with the virus at MOI 0.1. (D) qPCR shows upregulation of SARS-CoV-2 N1 in infected cells. (E) qPCR of inflammation markers CXCL12, NFKB1, and STAT3 show significant upregulation between mock and infected cells on day 3 of infection. Data is shown as mean \pm SEM with statistical significance determined by unpaired two-tailed t-test. ${ }^{\star} \mathrm{p}<0.05,{ }^{\star *} \mathrm{p}<0.01,{ }^{\star \star *} \mathrm{p}<0.001$. Scale bar represents $130 \mu \mathrm{m}$, and $10 \mu \mathrm{m}$ for zoomed panels adjacent to main images. ICC images shown here are representative results from 27 independent sites acquired. RNA was extracted from an aggregate of 6-16 biological replicates (using 96-well plates), and qPCR was run in 3 technical replicates per sample. These results were pooled from 2 independent rounds of infection experiments.

the higher variability in this subset of COVID-19 patients (Supplementary Figure 6). These results from COVID-19 and Control human tissues corroborate our novel results in iPan cell cultures, where we show that both pancreatic endocrine and exocrine cells can be infected by SARS-CoV-2, and the infection causes perturbations in pancreas-specific genes and the pancreatic cellular machinery.

\section{DISCUSSION}

In this novel study, by observing gene and protein expression in live human iPSC-derived pancreatic cultures and postmortem pancreatic tissue from COVID-19 patients, we have described the ability of SARS-CoV-2 to infect pancreatic cells. Both approaches indicated that endocrine islets and exocrine 
A

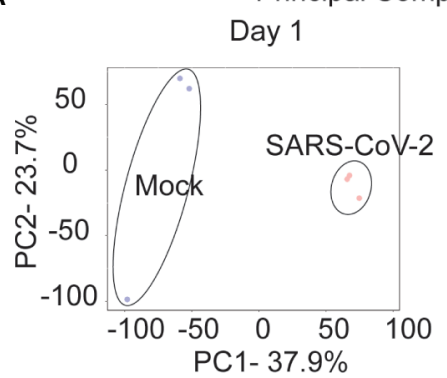

Principal Component Analysis

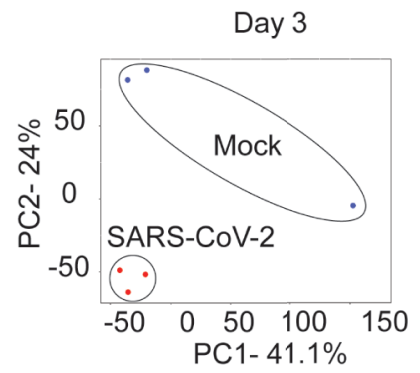

B

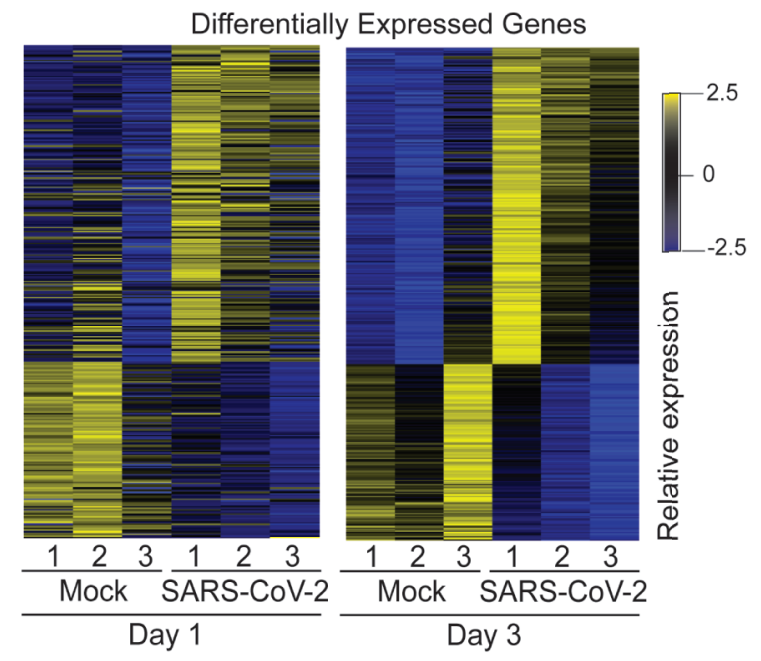

C

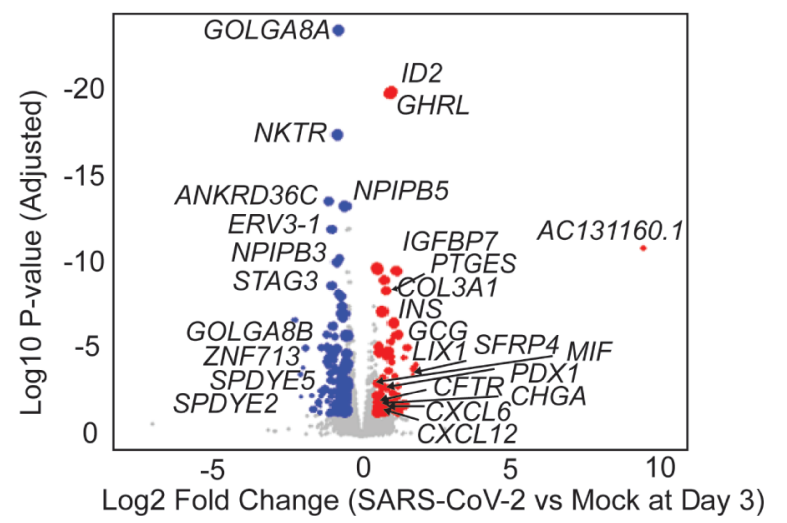

FIGURE 5 | RNAseq analysis of infected $\mathrm{Pan}^{\mathrm{EXO}}$ cells on days 1 and 3 post infection. (A) Principal Component Analysis of infected and mock samples collected on day 1 and day 3, respectively. (B) Differentially Expressed Genes Heat map showing the relative expression levels of transcripts differentially expressed with adjusted $p$-values less than 0.01. (C) Volcano plot of the log10 adjusted p-value of each expressed transcript on day 3 versus the log2 fold change. Transcripts that did not demonstrate differential expression with an adjusted $p$-value of less than 0.05 and a log2 fold-change in either direction greater than 0.5 are plotted in grey. Those that did were sized proportionally to their mean expression level, and genes of interest have been labeled.

acinar and ductal cells within the pancreas allow SARS-CoV-2 entry and establish infection. These cell types are known to express the ACE2 and TMPRSS2 transmembrane proteins recognized as the entry points for SARS-CoV-2 (Coate et al., 2020; Shang et al., 2020; Verdecchia et al., 2020; Müller et al., 2021). In our iPSC-derived pancreatic models, ACE2 and TMPRSS2 expression was observed in both acinar and ductal cells. These cultures also presented C-peptide ${ }^{+}$cells, and some of these endocrine cells expressed ACE2. Interestingly, recent clinical findings suggested pancreatic dysfunction following SARS-CoV-2 infection in COVID-19 patients (Apicella et al., 2020; Kusmartseva et al., 2020; Li et al., 2020; Liu et al., 2020). The iPSC-derived pancreatic cultures tested here were actively infected by SARS-CoV-2, as demonstrated by viral antigen expression and genomic replication. The cell culture observations were supported by similar results in 
A Enriched GO Biological Processes

Upregulated in Day 3 Infected Cells

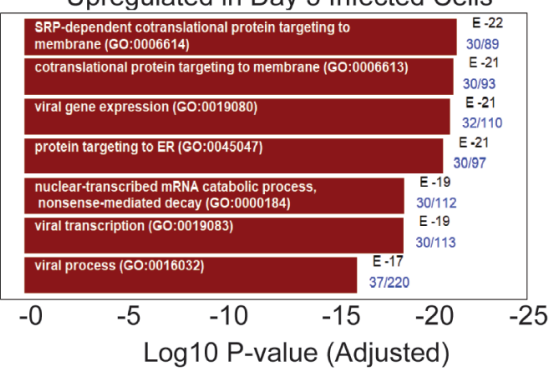

B

Enriched GO Cellular Component in Day 3 Infected Cells

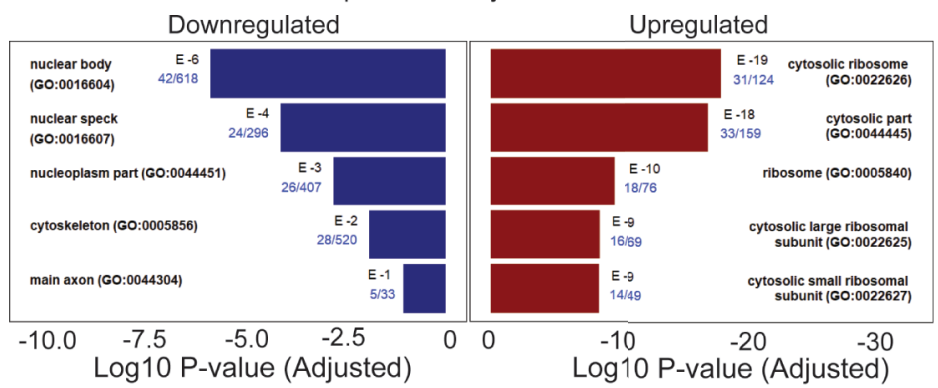

C Enriched COVID19-associated transcripts

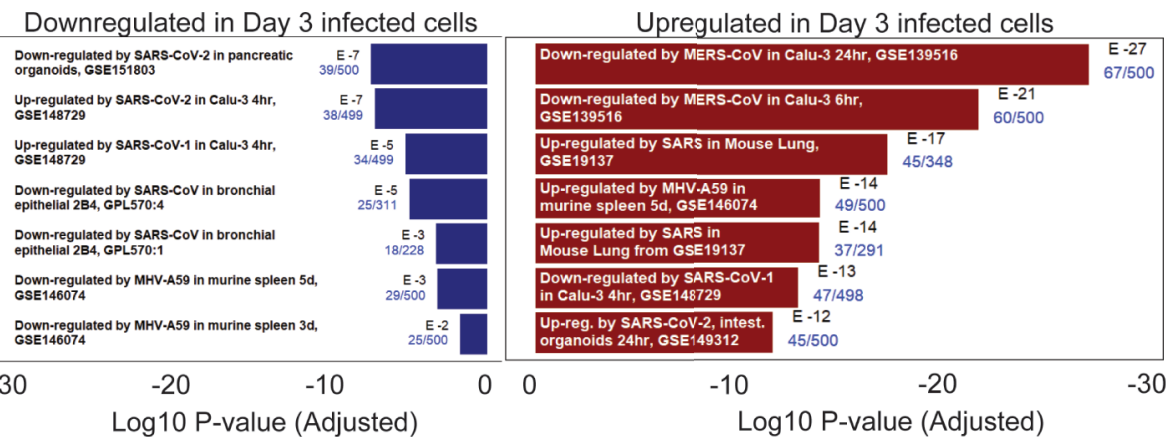

FIGURE 6 | RNAseq pathway and cellular component analysis of infected $\mathrm{Pan}{ }^{\mathrm{EXO}}$ cells on day 3 post infection. (A) Enriched GO Biological Processes: Upregulated biological processes were predicted based on the statistical overrepresentation of upregulated transcripts. The 500 most upregulated genes were submitted to Enrichr, and the Biological Processes in the Gene Ontology database which contained a statistically unlikely fraction of upregulated transcripts were plotted based on the probability of the observed enrichment. The fraction of genes in the gene set which were present in the 500 transcripts under analysis are listed in blue text. (B) Enriched GO Cellular Component: Upregulated and downregulated cellular components were predicted based on the statistical over representation of transcripts associated with these components. Nuclear bodies were predicted to be downregulated based on the presence of 42 out of 618 genes associated with this component in the list of 500 most downregulated transcripts in day 3 infected samples. Cytosolic ribosomes were predicted to be upregulated based on the presence of 31 out of 124 genes associated with this component among the 500 most upregulated transcripts on day 3 when compared to the Gene Expression Omnibus' cellular component gene sets (Barrett et al., 2013) (C) Enriched COVID19-associated transcripts: Gene sets identified as potentially similar to day 3 infected samples were plotted based on the statistical overrepresentation of the top 500 upregulated and downregulated transcripts.

post-mortem pancreatic tissues from COVID-19 patients. SARS-CoV-2-S protein was expressed in the pancreas of infected patients, specifically co-localizing in exocrine acinar cells and endocrine $\beta$-cells. Although we observed SARS-CoV$2-S$ protein expression in $\mathrm{CK} 19^{+}$ductal cells in culture and not in $\mathrm{CK}_{1} 9^{+}$cells from the limited set of post-mortem human pancreatic tissues of COVID-19 patients, a recent study did observe SARS-CoV-2-N protein expression in $\mathrm{CK} 19^{+}$ ductal cells in human tissues, suggesting some extent SARSCoV-2 infection is possible in human pancreatic ductal compartment (Müller et al., 2021). It is likely that there is some level of variability in susceptibility pancreatic ductal infection between patients that could be related to timing of virus infection their mortality. Interestingly, we also observed 
A

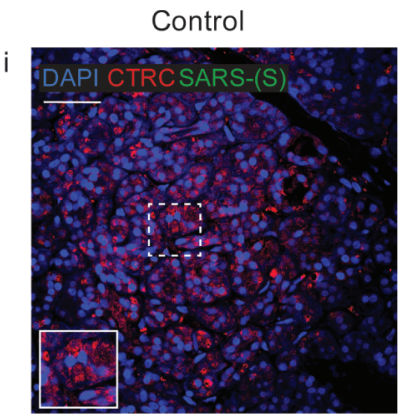

B

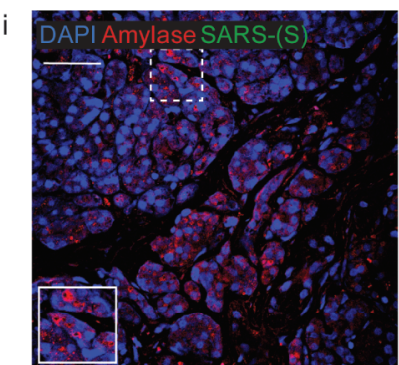

C

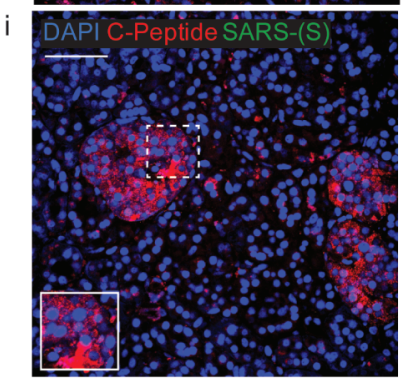

D

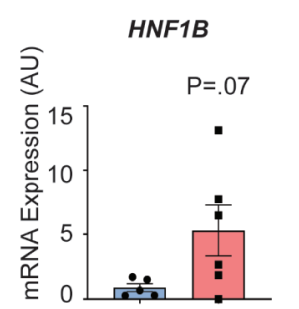

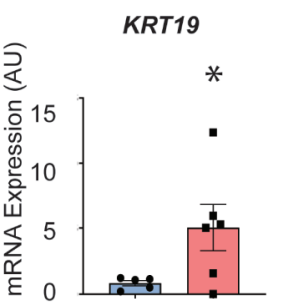
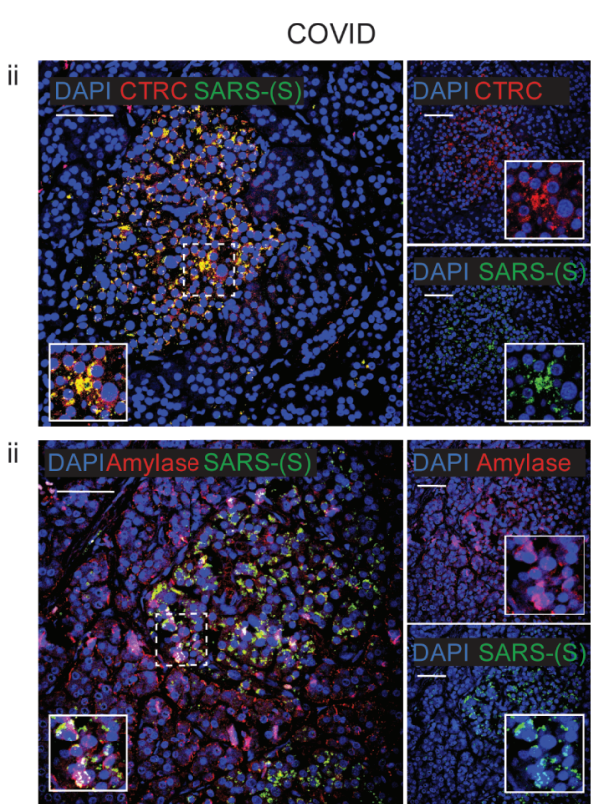

ii
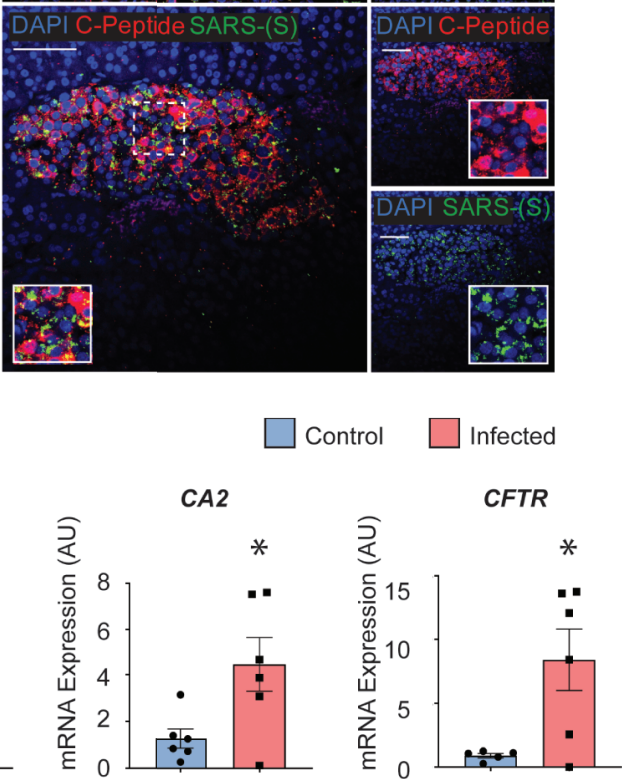

FIGURE 7 | Post-mortem human pancreas shows SARS-CoV-2 infectivity and co-localization with multiple pancreatic cell types. Immunohistochemistry results from pancreatic tissues of patients with COVID-19 show SARS-CoV-2-Spike S1 staining in green and pancreatic acinar markers in red, such as (A) ii. Chymotrypsin,

(B) ii. Amylase; and pancreatic endocrine marker (C) ii. C-peptide. (A-C) i. Corresponding sets of staining on non-COVID patients indicate no SARS-CoV-2-Spike S1 staining. (D) Pancreatic tissues from COVID-19 patients $(n=5)$ and Control subjects $(n=6)$ were utilized for RNA extraction. Real-time qPCR results show increased mRNA expression of ductal markers CA2, CFTR, KRT19 and CFTR. Data is shown as mean \pm SEM with statistical significance determined by unpaired two-tailed t-test. * $\mathrm{p}<0.05$. Scale bar represents $50 \mu \mathrm{M}$. IHC was performed in 3 non-COVID controls and 3 COVID patients. The results shown here are representative results from 3-10 independent sites acquired per patient. RNA was extracted from 6 non-COVID controls and 5 COVID patients, and qPCR was run in 3 technical replicates per sample.

SARS-CoV-2 infection in endocrine cells both in vitro and in post-mortem human tissues, and this was also observed by (Müller et al., 2021), where the infection of endocrine cells in vitro led to malfunction of cells and decreased glucosestimulated insulin secretion. This is notable given that diabetes and related complications are considered independent risk factors associated with higher mortality rates in COVID-19 (Holman et al., 2020).

Upon assessing cellular and molecular perturbations in iPSCderived pancreatic cultures, it was observed that SARS-CoV-2 infected ductal cells in culture were enlarged and the nuclear compartmentalization of the ductal-specific transcription factor 
SOX9, which is typically observed in normal ductal cells, was absent. Instead, a cytoplasmic staining pattern, suggesting a mislocalization of SOX9 as a potential indicator of cellular stress imposed by the virus in infected cells (Chakravarty et al., 2011). The elongated morphology of the infected ductal cells is also a feature that is common in cancerous or inflamed epithelial cells converting to fibrotic cells undergoing a dynamic process known as epithelial-mesenchymal transition (EMT) (Kalluri and Weinberg, 2009; Krantz et al., 2012). Furthermore, SARS-CoV-2 infected pancreatic cultures exhibit a large percentage of multinucleated fused $\mathrm{CK} 19^{+}$cells, a phenomenon described as syncytia formation; the fusion of multiple $\mathrm{ACE} 2^{+}$cells upon SARS-CoV-2 infection (Buchrieser et al., 2020). The histology of 41 lung samples from COVID-19 patients show that $87 \%$ of them display dysmorphic pneumocytes with syncytia (Bussani et al., 2020). In vitro studies have also shown syncytia formation in hiPSC-derived neurons, hiPSC-derived astrocytes, U2OSACE2, Vero-ACE2, and 293T-ACE2 after viral infection (Buchrieser et al., 2020; Wang et al., 2021; Zhang et al., 2021). Recent papers propose that a unique bi-arginine motif within the S1/S2 cleavage site of SARS-CoV-2 spike glycoprotein mediates the fusion of neighboring ACE2 ${ }^{+}$cells and formation of syncytia (Braga et al., 2021; Zhang et al., 2021). Furthermore, previous studies have found that syncytia mediated by SARS-CoV-2 as well as other viruses such as human immunodeficiency virus-1 (HIV-1) and reovirus-associated FAST proteins can lead to apoptosis (Scheller and Jassoy, 2001; Salsman et al., 2005; Nardacci et al., 2015; Buchrieser et al., 2020), This suggests that apoptosis in virus infected cultures becomes exacerbated via syncytia formation and cytopathy affects can be alleviated by targeting the relevant downstream processes specific to that cell type and virus. Pancreatic tissues from COVID-19 patients showed perturbation with upregulation of key ductal genes such as KRT19, CA2 and CFTR. Increased KRT19 expression is associated with pancreas-specific intracellular stress in patients with poor prognosis of pancreatic ductal adenocarcinoma (PDAC) (Yao et al., 2017). Cytoskeletal protein responses are known to be dysregulated under conditions of intracellular stress imposed by a viral infection and similar disturbances have also been reported to be an important in both immune and adaptive immunity (Kopecki et al., 2016; Ong et al., 2020) Yet, further elucidation of the mechanisms of pancreatic injury involved in response to the SARS-CoV-2 infection in pancreatic ductal cells is necessary. Granular morphology was noted in all SARS-CoV-2 positive cells and could be representative of viral replication and transportation occurring in membrane vesicles. This would be consistent with previous findings that SARS-CoV-2 associates with the host endo membrane system, with $40 \%$ of SARS-CoV-2interacting proteins having functions in the endomembrane system (Gordon et al., 2020).

In infected acinar cell cultures, the gene CXCL12 was upregulated. This gene, in particular, was found expressed tenfold in blood plasma by (Xu et al., 2020), and is consistent with the overexpression of similar cytokines and chemokines widely seen during the cytokine storm frequently observed in COVID19 patients (Coperchini et al., 2020; Song et al., 2020). Although
CXCL12 seems to promote tumor invasion, proliferation, angiogenesis, epithelial to mesenchymal transition and metastasis in pancreatic cancer (Righetti et al., 2019), its roles seem to be pleiotropic, and this could be due to the presence of at least six CXCL12 splicing isoforms, each with different roles (Righetti et al., 2019). To date, CXCL12 was reported to function as an anti-inflammatory chemokine during autoimmune inflammatory responses (Karin, 2010). In our SARS-CoV-2 infected iPSC-derived acinar cells, we believe CXCL12 could be playing either pro-inflammatory roles since other known proinflammatory transcription factors were also upregulated, such as NKFB1 and STAT3 (Ji et al., 2019), or a defensive antiinflammatory defensive to viral infection. The mechanistic role of CXCL12 upon SARS-CoV-2 infection of pancreatic cultures needs to be further investigated.

Upon probing the pathways disrupted by SARS-CoV-2 upon infection of pancreatic cells, the most dramatic transcriptional change was the over-expression of transcriptional machinery and SRP-dependent protein-targeting processes. The transcripts contributing to these pathways were identified as hallmarks of viral replication by gene enrichment analysis. It has been previously reported that a common feature of coronaviruses is the use of virus-engineered double membrane vesicles from host cell components as a central site for viral RNA synthesis (Snijder et al., 2020; Wolff et al., 2020). The upregulation of SRP-protein targeting processes could be a reflection of host cell machinery being repurposed for viral replication. Conversely, the top three downregulated cellular components were nuclear. Other coronaviruses, including avian infectious bronchitis virus (IBV) and murine hepatitis virus (MHV), and SARS-CoV have been found to arrest cell cycle in the nucleus, leading to increased viral replication (Chen and Makino, 2004; Dove et al., 2006; Yuan et al., 2006). Our results for the most upregulated and downregulated transcripts within the COVID-19-related Drug and Gene Set Library aligned with that of other coronaviruses, particularly SARS-CoV and MHV, with the top two downregulated transcripts being SARS-CoV-2 related. Taken all together, our results suggest that SARS-CoV-2, like other coronaviruses, elicits similar transcript-level signatures to promote viral replication in pancreatic cells. Future studies are necessary to confirm these mechanistic implications of these findings.

With this study, we provide novel patient-specific models for future mechanistic studies of SARS-CoV-2 impact on the pancreas. This study supports the utility of iPSC-derived pancreatic cells as an excellent platform to explore the detrimental impacts of SARS$\mathrm{CoV}-2$ on pancreatic cells. Further, detailed studies are required to better understand and perhaps the 3D organoids and Organ-Chip systems can be utilized to explore the short-term and long-term detrimental effects SARS-CoV-2 has on the pancreas.

\section{DATA AVAILABILITY STATEMENT}

The mRNA sequencing datasets presented in this study can be found in online repositories. The names of the repository/ 
repositories and accession number(s) can be found in the Methods section. Briefly, gene transcript tables as well as the original FASTQ files are available through NCBI's GEO database at accession number GSE165890.

\section{ETHICS STATEMENT}

Human cell lines, tissues and histology specimens were obtained or created at Cedars-Sinai under the auspices of the Cedars-Sinai Medical Center Institutional Review Board (IRB) approved protocols. Specifically, the iPSC cell lines and differentiation protocols in the present study were carried out in accordance with the guidelines approved by Stem Cell Research Oversight committee (SCRO) and IRB, under the auspices of IRB-SCRO Protocols Pro00032834 (iPSC Core Repository and Stem Cell Program) and Pro00036896 (Sareen Stem Cell Program). Infections of iPSC-derived cells were performed under the auspices of UCLA's Stem Cell Oversight Committee under protocol \#2020-004-01 and UCLA Biosafety Committee protocol BUA-2020-015-004-A. Post-mortem pancreatic tissues were collected by Cedars-Sinai's Biobank and Translational Research core in accordance with protocol Pro00036514, and lung tissues in accordance with the protocol Pro00035396. In vitro studies using human cell lines were conducted from participants that provided written informed consent for research studies. Remaining studies were conducted with post-mortem human specimens with appropriate IRB approvals.

\section{AUTHOR CONTRIBUTIONS}

SS: conception and design of the study, data collection, analysis, and interpretation, and manuscript writing. VW: conception and design of the study, data collection, analysis, and interpretation, and manuscript writing. RS: conception and design of the study, data collection, analysis, and interpretation, and manuscript writing. AG: data analysis and interpretation, and viral count analysis. YW: Next generation sequencing for mRNAsequencing experiments and data analysis. $\mathrm{HJ}, \mathrm{YZ}$, and $\mathrm{WH}$ : provision of study material (human pancreatic post-mortem tissues and slides). GG: SARS-CoV-2 infection of in vitro

\section{REFERENCES}

Amin M.. (2021). COVID-19 and the Liver: Overview. Eur. J. Gastroenterol. Hepatol. 68, 309-311. doi: 10.1097/MEG.0000000000001808

Apicella M., Campopiano M. C., Mantuano M., Mazoni L., Coppelli A., and Del Prato S. (2020) Covid-19 in People With Diabetes: Understanding the Reasons for Worse Outcomes. Lancet Diabetes Endocrinol. 8, 782-792. doi: 10.1016/S2213-8587(20)

Barrett T., Wilhite S. E., Ledoux P., Evangelista C., Kim I. F., Tomashevsky M., et al. (2013). Ncbi GEO: Archive for Functional Genomics Data Sets - Update.. Nucleic Acids Res. 41, 991-995 doi: 10.1093/nar/gks1193

Braga L., Ali H., Secco I., Chiavacci E., Neves G., Goldhill D., et al. (2021). Drugs That Inhibit TMEM16 Proteins Block SARS-CoV-2 Spike-Induced Syncytia. Nature. doi: 10.1038/s41586-021-03491-6 samples. VA: conception and design of the study, SARS-CoV-2 infection of in vitro samples. DS: conception and design of study, financial and administrative support, data analysis and interpretation, manuscript writing, and final approval of manuscript. All authors contributed to the article and approved the submitted version.

\section{FUNDING}

This work was supported by Cedars-Sinai Programmatic Funds to DS. This research was also funded by UCLA DGSOM and Broad Stem Cell Research Center institutional award (OCRC \#20-1) and (TRAN1COVID19-11975) to VA. The funders were not involved in the study design, collection, analysis, interpretation of data, the writing of this article or the decision to submit it for publication.

\section{ACKNOWLEDGMENTS}

We would like to acknowledge the support of the Cedars-Sinai Biomanufacturing Center facility and Cedars-Sinai Research Institute in supporting this project. We would like to thank The David and Janet Polak Foundation. The following reagents were obtained through BEI Resources, NIAID, NIH: Polyclonal Anti-SARS Coronavirus (antiserum, Guinea Pig), NR-10361; The following reagent was deposited by the Centers for Disease Control and Prevention and obtained through BEI Resources, NIAID, NIH: SARS-Related Coronavirus 2, Isolate USA-WA1/ 2020, NR-52281. We are grateful to Barbara Dillon, UCLA High Containment Program Director for BSL3 work. We would like to acknowledge Stephen Beil and Dr. Barry Stripp for kindly providing us human lung tissues (Lung Institute, Cedars-Sinai Medical Center, and to Aaron Frank (iPSC Core, Cedars-Sinai Medical Center) for kindly providing us human iPSC-derived neuron samples.

\section{SUPPLEMENTARY MATERIAL}

The Supplementary Material for this article can be found online at: https://www.frontiersin.org/articles/10.3389/fcimb.2021.678482/ full\#supplementary-material

Buchrieser J., Dufloo J., Hubert M., Monel B., Planas D., Rajah M. M., et al. (2020). Syncytia Formation by SARS-CoV-2-Infected Cells. EMBO J. 39, e106267. doi: 10.15252/embj.2020106267

Bussani R., Schneider E., Zentilin L., Collesi C., Ali H., Braga L., et al. (2020). Persistence of Viral RNA, Pneumocyte Syncytia and Thrombosis are Hallmarks of Advanced COVID-19 Pathology. EBioMedicine 61, 103104. doi: 10.1016/j.ebiom.2020.103104

Carithers L. J., Ardlie K., Barcus M., Branton P. A., Britton A., Buia S. A., et al. (2015). A Novel Approach to High-Quality Postmortem Tissue Procurement: The Gtex Project. Biopreserv. Biobank. 13, 311-317. doi: 10.1089/bio.2015.0032

Cevik M., Bamford C. G. G., and Ho A. (2020). Covid-19 Pandemic-A Focused Review for Clinicians. Clin. Microbiol. Infect. 26, 842-847. doi: 10.1016/ j.cmi.2020.04.023 
Chakravarty G., Moroz K., Makridakis N. M., Lloyd S. A., Galvez S. E., Canavello P. R., et al. (2011). Prognostic Significance of Cytoplasmic SOX9 in Invasive Ductal Carcinoma and Metastatic Breast Cancer. Exp. Biol. Med. 236, 145-155. doi: 10.1258/ebm.2010.010086

Chen C.-J., and Makino S. (2004). Murine Coronavirus Replication Induces Cell Cycle Arrest in G0/G1 Phase. J. Virol. 78, 128. doi: 10.1128/JVI.78.11.56585669.2004

Chen E. Y., Tan C. M., Kou Y., Duan Q., Wang Z., Meirelles G. V., et al. (2013). Enrichr: Interactive and Collaborative HTML5 Gene List Enrichment Analysis Tool. BMC Bioinf. 14. doi: 10.1186/1471-2105-14-128

Coate K. C., Cha J., Shrestha S., Wang W., Gonçalves L. M., Almaça J., et al. (2020). SARS-Cov-2 Cell Entry Factors ACE2 and TMPRSS2 are Expressed in the Pancreas But are Not Enriched in Islet Endocrine Cells. BioRxiv Prepr. Serv. Biol. doi: 10.1101/2020.08.31.275719

Coperchini F., Chiovato L., Croce L., Magri F., and Rotondi M. (2020). The Cytokine Storm in COVID-19: An Overview of the Involvement of the Chemokine/Chemokine-Receptor System. Cytokine Growth Factor Rev. 53, 25-32. doi: 10.1016/j.cytogfr.2020.05.003

Dataset (2020). GitHub - CSSEGISandData/COVID-19: Novel Coronavirus (COVID-19) Cases, provided by JHU CSSE.

de-Madaria E., and Capurso G. (2021). Covid-19 and Acute Pancreatitis: Examining the Causality. Nat. Rev. Gastroenterol. Hepatol. 18, 3-4. doi: 10.1038/s41575-020-00389-y

Desjardins P, and Conklin D. (2010) NanoDrop Microvolume Quantitation of Nucleic Acids. J. Vis. Exp. (45), 2565. doi: 10.3791/2565

Dobin A., Davis C. A., Schlesinger F., Drenkow J., Zaleski C., Jha S., et al. (2013). Star: Ultrafast Universal RNA-seq Aligner. Bioinformatics 29, 15-21. doi: 10.1093/bioinformatics/bts635

Dove B., Brooks G., Bicknell K., Wurm T., and Hiscox J. A. (2006). Cell Cycle Perturbations Induced by Infection With the Coronavirus Infectious Bronchitis Virus and Their Effect on Virus Replication. J. Virol. 80, 41474156. doi: 10.1128/JVI.80.8.4147-4156.2006

Gordon D. E., Jang G. M., Bouhaddou M., Xu J., Obernier K., White K. M., et al. (2020). A SARS-CoV-2 Protein Interaction Map Reveals Targets for Drug Repurposing. Nature 583, 459-468. doi: 10.1038/s41586-020-2286-9

Hoffmann M., Kleine-Weber H., Schroeder S., Krüger N., Herrler T., Erichsen S., et al. (2020). SARS-Cov-2 Cell Entry Depends on ACE2 and TMPRSS2 and Is Blocked by a Clinically Proven Protease Inhibitor. Cell 181, 271-280. e8. doi: 10.1016/j.cell.2020.02.052

Hohwieler M., Illing A., Hermann P. C., Mayer T., Stockmann M., Perkhofer L., et al. (2017). Human Pluripotent Stem Cell-Derived Acinar/Ductal Organoids Generate Human Pancreas Upon Orthotopic Transplantation and Allow Disease Modelling. Gut 66, 473-486. doi: 10.1136/gutjnl-2016-312423

Holman N., Knighton P., Kar P., O’Keefe J., Curley M., Weaver A., et al. (2020). Risk Factors for COVID-19-related Mortality in People With Type 1 and Type 2 Diabetes in England: A Population-Based Cohort Study. Lancet Diabetes Endocrinol. 8, 823-833. doi: 10.1016/S2213-8587(20)30271-0

Ji Z., He L., Regev A., and Struhl K. (2019). Inflammatory Regulatory Network Mediated by the Joint Action of NF-kB, STAT3, and AP-1 Factors is Involved in Many Human Cancers. Proc. Natl. Acad. Sci. USA 116, 9453-9462. doi: $10.1073 /$ pnas.1821068116

Kalluri R., and Weinberg R. A. (2009). The Basics of Epithelial-Mesenchymal Transition. J. Clin. Invest. 119, 1420-1428. doi: 10.1172/JCI39104

Karin N. (2010). The Multiple Faces of CXCL12 (Sdf-1 $\alpha$ ) in the Regulation of Immunity During Health and Disease. J. Leukoc. Biol. 88, 463-473. doi: 10.1189/jlb.0909602

Kopecki Z., Ludwig R. J., and Cowin A. J. (2016). Cytoskeletal Regulation of Inflammation and its Impact on Skin Blistering Disease Epidermolysis Bullosa Acquisita. Int. J. Mol. Sci. 17, 1116. doi: 10.3390/ijms17071116

Kosti I., Jain N., Aran D., Butte A. J., and Sirota M. (2016). Cross-Tissue Analysis of Gene and Protein Expression in Normal and Cancer Tissues OPEN. Nat. Publ. Gr. 6, 24799. doi: 10.1038/srep24799

Krantz S. B., Shields M. A., Dangi-Garimella S., Munshi H. G., and Bentrem D. J. (2012). Contribution of Epithelial-to-Mesenchymal Transition and Cancer Stem Cells to Pancreatic Cancer Progression. J. Surg. Res. 173, 105-112. doi: 10.1016/j.jss.2011.09.020
Kuleshov M. V., Jones M. R., Rouillard A. D., Fernandez N. F., Duan Q., Wang Z., et al. (2016). Enrichr: A Comprehensive Gene Set Enrichment Analysis Web Server 2016 Update. Nucleic Acids Res. 44, W90-W97. doi: 10.1093/nar/gkw377

Kuleshov M. V., Stein D. J., Clarke D. J. B., Kropiwnicki E., Jagodnik K. M., Bartal A., et al. (2020). The COVID-19 Drug and Gene Set Library. Patterns 1. doi: 10.1016/j.patter.2020.100090

Kumaran N. K., Karmakar B. K., and Taylor O. M. (2020). Coronavirus disease-19 (Covid-19) Associated With Acute Necrotising Pancreatitis (ANP). BMJ Case Rep. 13, e237903. doi: 10.1136/bcr-2020-237903

Kusmartseva I., Wu W., Syed F., van der Heide V., Jorgensen M., Joseph P., et al. (2020). Expression of SARS-CoV-2 Entry Factors in the Pancreas of Normal Organ Donors and Individuals With COVID-19. Cell Metab. 32, 10411051.e6. doi: 10.1016/j.cmet.2020.11.005

Le Sage V., and Mouland A. J. (2013). Viral Subversion of the Nuclear Pore Complex. Viruses 5, 2019-2042. doi: 10.3390/v5082019

Li B., and Dewey C. N. (2011). Rsem: Accurate Transcript Quantification From RNA-Seq Data With or Without a Reference Genome. BMC Bioinf. 12, 323. doi: 10.1186/1471-2105-12-323

Li M. Y., Li L., Zhang Y., and Wang X. S. (2020). Expression of the SARS-CoV-2 Cell Receptor Gene ACE2 in a Wide Variety of Human Tissues. Infect. Dis. Poverty 9, 45. doi: 10.1186/s40249-020-00662-x

Liu F., Long X., Zhang B., Zhang W., Chen X., and Zhang Z. (2020). Ace2 Expression in Pancreas may Cause Pancreatic Damage After SARS-CoV-2 Infection. Clin. Gastroenterol. Hepatol. 18, 2128-2130.e2. doi: 10.1016/j.cgh.2020.04.040

Lonsdale J., Thomas J., Salvatore M., Phillips R., Lo E., Shad S., et al. (2013). The Genotype-Tissue Expression (Gtex) Project. Nature Genet doi: 10.1038/ ng.2653

Memon B., Karam M., Al-Khawaga S., and Abdelalim E. M. (2018). Enhanced Differentiation of Human Pluripotent Stem Cells Into Pancreatic Progenitors Co-Expressing PDX1 and NKX6.1. Stem Cell Res. Ther. 9, 15. doi: 10.1186/ s13287-017-0759-z

Mukherjee R., Smith A., and Sutton R. (2020). Covid-19-related Pancreatic Injury. Br. J. Surg. 107, e1900. doi: 10.1002/bjs.11645

Müller J. A., Groß R., Conzelmann C., Krüger J., Merle U., Steinhart J., et al. (2021). Sars-CoV-2 Infects and Replicates in Cells of the Human Endocrine and Exocrine Pancreas. Nat. Metab. 3, 149-165. doi: 10.1038/s42255-021-00347-1

Nardacci R., Perfettini J., Grieco L., Thieffry D., Kroemer G., and Piacentini M. (2015). Syncytial Apoptosis Signaling Network Induced by the HIV-1 Envelope Glycoprotein Complex: An Overview. Cell Death and Dis 6, e1846. doi: 10.1038/cddis.2015.204

Ong M. S., Deng S., Halim C. E., Cai W., Tan T. Z., Huang R. Y. J., et al. (2020). Cytoskeletal Proteins in Cancer and Intracellular Stress: A Therapeutic Perspective. Cancers (Basel) 12, 238. doi: 10.3390/cancers 12010238

Pelayo J., Lo K. B., Bhargav R., Gul F., Peterson E., Dejoy R., et al. (2020). Clinical Characteristics and Outcomes of Community- and Hospital-Acquired Acute Kidney Injury With COVID-19 in a US Inner City Hospital System. Cardio Renal Med. 10, 223-231. doi: 10.1159/000509182

Pinte L., and Baicus C. (2020). Pancreatic Involvement in SARS-CoV-2: Case Report and Living Review. J. Gastrointestin. Liver Dis. 29, 277-276. doi: $10.15403 /$ jgld-2618

Rajamani U., Gross A. R., Hjelm B. E., Sequeira A., Vawter M. P., Tang J., et al. (2018). Super-Obese Patient-Derived Ipsc Hypothalamic Neurons Exhibit Obesogenic Signatures and Hormone Responses. Cell Stem Cell 22, 698712.e9. doi: 10.1016/j.stem.2018.03.009

Rawla P., Bandaru S. S., and Vellipuram A. R. (2017). Review of Infectious Etiology of Acute Pancreatitis. Gastroenterol. Res. 10, 153-158. doi: 10.14740/gr858w

Righetti A., Giulietti M., Šabanović B., Occhipinti G., Principato G., and Piva F. (2019). CXCL12 and Its Isoforms: Different Roles in Pancreatic Cancer? J. Oncol. 2019. doi: 10.1155/2019/9681698

Salsman J., Top D., Boutilier J., and Duncan R. (2005). Extensive Syncytium Formation Mediated by the Reovirus Fast Proteins Triggers ApoptosisInduced Membrane Instability. J. Virol. 79, 8090-8100. doi: 10.1128/ JVI.79.13.8090-8100.2005

Scheller C., and Jassoy C. (2001). Syncytium Formation Amplifies Apoptotic Signals: A New View on Apoptosis in HIV Infection In Vitro. Virology 282, 48 55. doi: 10.1006/viro.2000.0811 
Shang J., Wan Y., Luo C., Ye G., Geng Q., Auerbach A., et al. (2020). Cell Entry Mechanisms of SARS-CoV-2. Proc. Natl. Acad. Sci. USA 117, 11727-11734. doi: $10.1073 /$ pnas.2003138117

Sharma A., Garcia G., Wang Y., Plummer J. T., Morizono K., Arumugaswami V., et al. (2020). Human Ipsc-Derived Cardiomyocytes are Susceptible to SARS-CoV-2 Infection. Cell Rep. Med. 1, 100052. doi: 10.1016/j.xcrm.2020.100052

Snijder E. J., Limpens R. W. A. L., de Wilde A. H., de Jong A. W. M., Zevenhoven-Dobbe J. C., Maier H. J., et al. (2020). A Unifying Structural and Functional Model of the Coronavirus Replication Organelle: Tracking Down RNA Synthesis. PloS Biol. 18, e3000715. doi: 10.1371/journal.pbio.3000715

Song P., Li W., Xie J., Hou Y., and You C. (2020). Cytokine Storm Induced by SARS-CoV-2. Clin. Chim. Acta 509, 280-287. doi: 1016/j.cca.2020.06.017

Tuttolomondo D., Frizzelli A., Aiello M., Bertorelli G., Majori M., and Chetta A. (2020). Beyond the Lung Involvement in COVID-19 Patients. A Review. Minerva Med. doi: 10.23736/S0026-4806.20.06719-1

Varga Z., Flammer A. J., Steiger P., Haberecker M., Andermatt R., Zinkernagel A. S., et al. (2020). Endothelial Cell Infection and Endotheliitis in COVID-19. Lancet 395, 1417-1418. doi: 10.1016/S0140-6736(20)30937-5

Verdecchia P., Cavallini C., Spanevello A., and Angeli F. (2020). The Pivotal Link Between ACE2 Deficiency and SARS-CoV-2 Infection. Eur. J. Intern. Med. 76, 14-20. doi: 10.1016/j.ejim.2020.04.037

Wang C., Zhang M., Garcia G., Tian E., Cui Q., Chen X., et al. (2021). ApoeIsoform-Dependent SARS-CoV-2 Neurotropism and Cellular Response. Cell Stem Cell 28, 331-342.e5. doi: 10.1016/j.stem.2020.12.018

Wolff G., Melia C. E., Snijder E. J., and Bárcena M. (2020). Double-Membrane Vesicles as Platforms for Viral Replication. Trends Microbiol. 28, 1022-1033. doi: 10.1016/j.tim.2020.05.009
Xu Z. S., Shu T., Kang L., Wu D., Zhou X., Liao B. W., et al. (2020). Temporal Profiling of Plasma Cytokines, Chemokines and Growth Factors From Mild, Severe and Fatal COVID-19 Patients. Signal Transduction Targeting Ther. 5, 100. doi: 10.1038/s41392-020-0211-1

Yao H., Yang Z., Liu Z., Miao X., Yang L., Li D., et al. (2017). Glypican-3 and KRT19 are Markers Associating With Metastasis and Poor Prognosis of Pancreatic Ductal Adenocarcinoma. Cancer Biomarkers 17, 397-404. doi: 10.3233/CBM-160655

Yuan X., Wu J., Shan Y., Yao Z., Dong B., Chen B., et al. (2006). SARS Coronavirus 7a Protein Blocks Cell Cycle Progression at G0/G1 Phase Via the Cyclin D3/ pRb Pathway. Virology 346, 74-85. doi: 10.1016/j.virol.2005.10.015

Zhang Z., Zheng Y., Niu Z., Zhang B., Wang C., Yao X., et al. (2021). Sars-CoV-2 Spike Protein Dictates Syncytium-Mediated Lymphocyte Elimination. Cell Death Differ. 1-3. doi: 10.1038/s41418-021-00782-3

Conflict of Interest: The authors declare that the research was conducted in the absence of any commercial or financial relationships that could be construed as a potential conflict of interest.

Copyright (C) 2021 Shaharuddin, Wang, Santos, Gross, Wang, Jawanda, Zhang, Hasan, Garcia, Arumugaswami and Sareen. This is an open-access article distributed under the terms of the Creative Commons Attribution License (CC BY). The use, distribution or reproduction in other forums is permitted, provided the original author(s) and the copyright owner(s) are credited and that the original publication in this journal is cited, in accordance with accepted academic practice. No use, distribution or reproduction is permitted which does not comply with these terms. 